\title{
Fingerprints, Iris and DNA Features based Multimodal Systems: A Review
}

\author{
Prakash Chandra Srivastava1, Anupam Agrawal ${ }^{2}$, Kamta Nath Mishra ${ }^{*}$, P. K. Ojha ${ }^{3}$, R. Garg ${ }^{4}$ \\ $1,1^{*}, 3,4$ Department of Computer Science \& Engg., Birla Institute of Technology, Ranchi (Alld. Campus), INDIA \\ ${ }^{2}$ Department of Information Technology, Indian Institute of Information Technology, Allahabad, U. P., INDIA \\ Email: prakash_bit123@rediffmail.com, anupam69@gmail.com, mishrakn@yahoo.com, \\ pradeepkumarojha@yahoo.com,rishug18@gmail.com
}

\begin{abstract}
Biometric systems are alternates to the traditional identification systems. This paper provides an overview of single feature and multiple features based biometric systems, including the performance of physiological characteristics (such as fingerprint, hand geometry, head recognition, iris, retina, face recognition, DNA recognition, palm prints, heartbeat, finger veins, palates etc) and behavioral characteristics (such as body language, facial expression, signature verification, speech recognition, Gait Signature etc.).

The fingerprints, iris image, and DNA features based multimodal systems and their performances are analyzed in terms of security, reliability, accuracy, and long-term stability. The strengths and weaknesses of various multiple features based biometric approaches published so far are analyzed. The directions of future research work for robust personal identification is outlined.
\end{abstract}

Index Terms - DNA Sequence, Fingerprint Patterns, Iris Pattern, Multimodal Systems, Palates

\section{Introduction}

According to International Standard Organization (ISO), biometric means "automated recognition of individuals on the basis of their physiological and behavioral characteristics". A biometric system is also known as human recognizer or human identifier or human authenticator. Every human being can be uniquely identified on the basis of physiological \& behavioral characteristics ${ }^{[104][75]}$. The term biometric is defined from two Greek words 'bio' means life and 'metric' means measurement. In 500 B.C. Babylonians used fingerprints for business transactions. In the same period Egyptians used hand print image to differentiate between trusted and un-trusted traders.

Corresponding Author: Kamta Nath Mishra, Assistant Professor, Department of Computer Science \& Engg., Birla Institute of Technology, Mesra, Ranchi, INDIA (Allahabad Campus), Email: mishrakn@yahoo.com, PhoneNo.:0091-9695052989
Chinese and Romans used hand image for business transaction and for identify ing family members almost in the same period. In the mid of 1800's French people started using Bertillon system based methods for body dimension measurement. The first fingerprint patterns and ridges based biometric system was developed in India by Azizul Haque for Ed ward Henry. Th is is called Henry's system ${ }^{[99]}$.

The different biometric aspects used for personal identification are: fingerprints, face, hand geometry, palm print, heartbeat, finger veins, gait signature, palates, DNA recognition, iris, facial expression, body language and voice etc. Fingerprint recognition involves capturing the images of fingerprints and storing them in database. The properties of ridges, cores, deltas, whorls, and minutiae points can provide a unique identification pattern ${ }^{[104][75][38]}$.

Face is directly associated with identity, gender, and age group of a person. Face recognition comprises of two aspects ${ }^{[40]}$ :

Aspect 1: Face Appearance (In face appearance, the basic aspects are attractiveness, age, face structure, and facial skin)

Aspect 2: Facial Expression (The facial expression changes with change in age of a person. Facial expression includes pose, and changes in face expression)

The hand geometry based systems measure a user's hand in many dimensions e.g. hand length, finger length, finger width, palm length, and palm width. The CCD camera reads the hand shape by recording the silhouettes and these silhouettes are used for identifying a person. In palm prints based identification methods the palm print image of a person is captured by CCD camera and it is compared with the actual image stored in the database. This matching procedure is based on palm characteristics (principle lines, wrinkles, ridges, and minutiae points $)^{[104][67]}$. Every human has a heart that keeps on pumping for whole life. Electrocardiograms (ECG) are used to record the electrical activity of the heart. The digital images of heartbeats can be captured using an electronic stethoscope. The digitized heartbeat frequencies can be 
used for online personal identification. However it may not be possible to identify the persons suffering from low blood pressure / high blood pressure using heartbeat frequencies ${ }^{[71]}$.

In finger veins based identification method, the internal structure of finger veins are scanned using a scanner and this internal structure of finger veins are used for online identification of a person ${ }^{[76]}$. Gait signatures are the images or impressions left behind by a walking person. Gait signature is based on sensor measurement. Sensor determines the pressure sensitivity on the floor. The sensor material is called Electro Mechanical Film (EMF). The EMF uses the concept of unique walking style of a person for identification ${ }^{[96][56]}$. Palatal rugae patterns are the lines available on the surface of a palate. These lines are unique for each person and therefore, we can use palatal rugae patterns for identifying a person. The study of palatal patterns for identifying an individual is named as palatoscopy or palatal rugoscopy ${ }^{[50][12]}$.

Deoxyribonucleic Acid (DNA) has a double helix structure and it gives the most reliable result for offline personal identification excluding identical twins cases. Every human has a unique DNA sequence excluding identical twins. The fraternal twins have different DNA sequences and identical twins have exactly same DNA sequence. DNA sequence consists of four alphabets namely: Adenine (A), Guanine (G), Cytosine $(\mathrm{C})$, and Thymine $(\mathrm{T})$. The unknown nucleotide $(\mathrm{N})$ present in a DNA sequence can be either Adenine or Guanine or Cytosine or Thymine ${ }^{[64]}$. An iris is obtained after removing pupil, eye brow, skin and other noise disturbances from the eye of a person. The iris image consists of Red (R), Green (G), and Blue (B) colors and each person including identical has a unique iris. Therefore, an iris based system can be considered as a reliable and secure system for personal identification ${ }^{[23]}$. In voice based recognition techniques the input voice is captured by voice processing machine and the machine uniquely identifies a person on the basis of voice characteristics e.g. frequency, inflection, flow, and cadence etc ${ }^{[16]}$. The facial expressions of a person can be easily mimicked by another person. Therefore, it should be combined with body language and voice for identifying a person. A combination of two or more physiological and behavioral features of a person can also be used for personal identification.

Biometric systems verify the identity of a person with respect to physiological and behavioral features [48][75]. Some of the physiological and behavioral characteristics used for personal identification are presented in figure 1. Consider a set that takes all biometric identifiers including Hand Writing (HW), Hand Geometry $(H G)$, Ear Geometry $(E G)$, Iris Image $(I I)$, Lip Motion $(L P)$, Palatal Structure $(P S)$, Heart Beat $(H B)$, Palm Print $(P P)$, Finger Print $(F P)$, Finger Veins $(F V)$, Body Expression $(B E)$, DNA Sequence $(D S)$, Thump Print $(T P)$, Voice Recognition $(V R)$, Head Movement $(H M)$, Face Structure $(F S)$ and Gait Signature
(GS). Therefore, a Single Biometric System (SBS) can be defined by following mathematical expression:

$S B S=\{H W, H G, E G, I I, L P, P S, H B, P P, F P, F V$,

$B E, D S, T P, V R, H M, F S, G S\}$

Here, $\left(b_{1}, b_{2}, b_{3}, b_{4}, . . b_{n}\right\} \in S B S$, where, $b_{\mathrm{i}}$ is a single biometric identifier. Hence, $M=b_{1} \vee b_{2} \vee b_{3} \vee b_{4} \vee \ldots$ $\vee b_{n}$, where ' $\mathrm{M}$ ' will represent a single biometric identifier and it is insufficient for foolproof personal identification ${ }^{[40]}$.

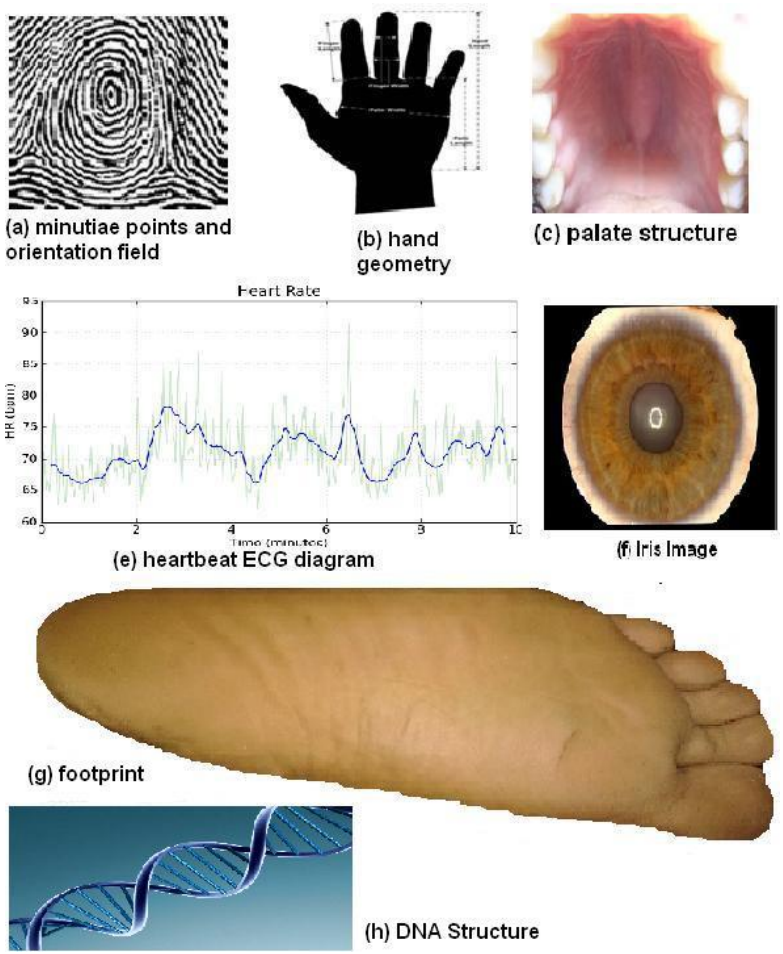

Fig. 1: Physiological and behavioral features used for personal identification

An expert person can copy gait signature, handwriting, voice, body language, and facial expressions of another person. Identical twins have the same face structure. Two persons may have same pal print, hand geometry, and finger veins but each person has unique fingerprints, iris image, and DNA sequence excluding identical twins. An identical twin pair may differ in fingerprints and iris images but they may share the same DNA sequence. The fingerprints and irises decompose very soon after the death of a person but the DNA sequence never decomposes. Thus, it is always important to use fingerprints, iris image, and DNA features based multimodal system for identifying a dead and an alive person. Hence, we have reviewed and analyzed the performance of fingerprints, iris, and DNA features based multimodal systems in this research work.

In section 2 of this research work we have discussed the background of physiological and behavioral systems. The review, classification, and analysis of fingerprint based systems are discussed in section 3. The survey and analysis of iris based systems are 
discussed in section 4. Compression and decompression of DNA sequences are discussed in section 5. The classification and analysis of multimodal systems are discussed in section 6. The conclusion and future directions of multimodal systems are discussed in section 7 .

\section{Background of Biometric Systems}

The biometric systems are classified into two categories: physiological features based biometrics and Behavioral features based biometrics.

\subsection{What is a Physiological Feature?}

A physiological feature is a feature which is physically present in the body of a person. These features are extracted from human body by using specific equipments and techniques.

\subsubsection{Physiological Features Based Biometrics}

The physiological features based biometrics include iris image, finger prints, thumb print, palm print, face, finger veins, hand geometry, DNA sequence, iris image, heartbeat, and palates etc.

Face recognition system analyzes the face on static images and video-based approaches. Age is an important attribute of human face. Identification and verification problems may be solved by using face recognition systems. Face recognition is used as a personal identification method in the areas of multimedia security, home security, and searching for missing individuals [96]. Face based recognition techniques store face images through a high definition digital video camera and uniquely identify a persons on the basis of face metric and eigen face properties. A human face can be easily altered through surgery or mask. The face of a person changes from childhood to become old age citizen ${ }^{[53]}$. Therefore, face recognition based system is not a robust system for personal identification. In illness, the voice of a person may change and hence voice based biometric system is also not a foolproof system.

Iris is used for personal identification in highly secured zones e.g. nuclear reactors. An iris image contains a large number of visible pixel patterns which are unique for an individual and these patterns remain stable with age. The left and right irises are different for the same person. Iris recognition system includes image acquisition, iris localization, and matching iris patterns. Iris Recognition method scans the iris image with the help of digital camera and authenticates a person on the basis of iris characteristics. In retina based identification the retina image is captured using infrared scanning technique and this method uniquely identifies a person on the basis of retina characteristics e.g. image of the blood vessels ${ }^{[59]}$.

An interactive image enhancement technique based on fuzzy relaxation was presented by Zhou which can be used for enhancing fingerprints and iris images ${ }^{[107]}$. Fingerprint based identification is one of the most important identification techniques. Specific fingerprints can be identified by the patterns of ridges, furrows, and minutiae points. Veins patterns or vascular patterns remain same until the death of a person. Veins of the palm are unique for every individual and apart from size; this pattern will not vary over the course of a person's lifetime. Therefore, palm-vein patterns based biometric systems are used for secure personal identification ${ }^{[76]}$.

The decomposed bodies and burnt bodies are special cases where fingerprints are not available. In these cases we can use palates based methods or DNA sequence matching for identification. Hu man ear based biometric systems have been used for many years to identify a person in forensic laboratories. The demining space between specific points of the ear can be used for personal identification. This method uses image normalization, contour detection, binarization, coordinates normalization, and geometrical feature extraction steps for personal identification ${ }^{[10]}$.

Hand Geometry based identity verification techniques are implemented using the characteristics of hand geometry (length of hand, length of finger, width of finger, structure of finger, thickness of palm, and width of palm) ${ }^{[55]}$. Palate based biometric systems can be used for personal identification. Palates don't change during the life of a person. Palates are unique for each individual and remain stable during the growth of a person. Palate is used in the cases where fingerprints are not available ${ }^{[78]}$.

\subsubsection{Limitations of Physiological Features Based Biometrics}

The degradation of fingerprint caused by occupation is a disadvantage of finger print based identification. The fingerprints may be altered or modified by using certain unfair means approaches. Hand geometry based identification system has low accuracy because two or more persons may have similar hand geometry ${ }^{[55]}$. Iris based identification is a secure biometric system but the acquisition of an iris image requires more training and it is difficult to capture the correct iris image ${ }^{[38]}$. The DNA sequence based identification system cannot be used for online identification. Identical twins may have same DNA sequence. Finger veins based biometric identification systems are not applicable for identifying a handicapped person. Heartbeat based identification system may fail for identify ing a person with abnormal blood pressure. Body expressions cannot be used for identifying a person because a person can easily copy the body language of another person. In palm print based identification methods, the area of palm doesn't remain same during the life period and duplicity may exist between palm prints of two persons. Identifying a person using ear geometry based identification system has very high false acceptance rate percentage. 


\subsection{What is a Behavioral Feature?}

The behavioral features are the features which are extracted from day-to-day sociological behaviors of a person.

\subsubsection{Behavioral Features Based Biometrics}

The behavioral features used for personal identification are gait signature, voice, lip motion, body language, and handwriting etc. Gait signatures are used to identify an individual. In this identification method the system captures gait signature with the help of a high resolution digital camera. The acquired gait signature is converted into grayscale image. The features of gait signatures are extracted from the image and are online compared with stored gait signatures of database. On the basis of matching results a person is either accepted or rejected by the system ${ }^{[96]}$.

Table 1: Performance of Face, Signature, heartbeat, palate, voice, and body language with lip motion based automated systems ${ }^{[10][12][41]}$.

\begin{tabular}{cccccccc}
\cline { 2 - 7 } & & & & & & $\begin{array}{c}\text { Body } \\
\text { Language } \\
\text { with Lip } \\
\text { Motion }\end{array}$ \\
\hline Universality & High & Low & Low & High & Medium & Low \\
\hline Uniqueness & Low & Low & Low & High & Low & Low \\
\hline Permanence & Medium & Low & Low & High & Low & Low \\
\hline Collectability & High & High & High & High & Medium & Low \\
\hline Performance & Low & Low & Low & High & Low & Low \\
\hline Acceptability & High & High & Low & Medium & High & Low \\
\hline Circumvention & Low & Low & Low & High & Low & Low \\
\hline FAR & Medium & Low & High & Low & Low & High \\
\hline FRR & Medium & Low & High & Low & High & High \\
\hline
\end{tabular}

Today, many biometric technologies are available. Amongst these technologies, voice based identity validation has a unique edge. The human voice is unique because of physiological and behavioral aspects of speech production. Every human has unique voice. The human voice includes numerous characteristics e.g. cadence, frequency, pitch, and tone. Every person has unique voice frequency, tone, pitch, and cadence. Voice based biometric solutions create a voice print which is a template of the characteristics of unique voice. A particular person can be identified on the basis of these unique features of voice. Voice recognition system can be classified into two categories ${ }^{[10]}$ :

Category 1: Text-Dependent (In text-dependent method, the person will have to read predefined words, sentences, and specific phrases specified in the system)
Category 2: Text-Independent (In text-independent method, the person can speak anything which he wants to speak)

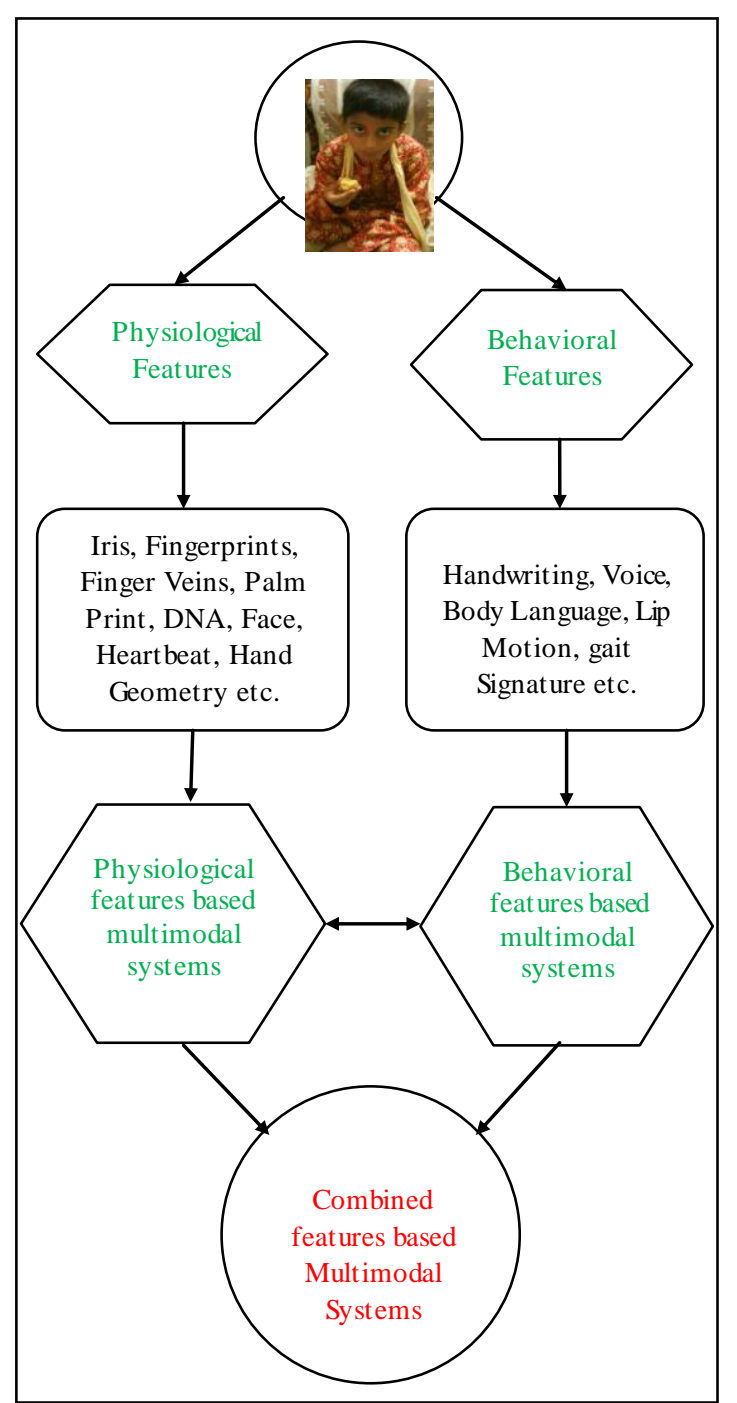

Fig. 2: Deriving Multimodal System from Physiological and Behavioral Characteristics of a person

The online voice sample is compared with prerecorded templates. A person's voice changes overtime with the physical growth and it may also change due to coldness or specific diseases. High level voice matching methods use accents, talking style, and dialects for identifying a person ${ }^{[10]}$.

The Performance of Face, Signature, Heart Beat, Palate, Voice, and Body Language with Lip Motion based automated systems on a software scale are shown in table 1 . The results of table 1 show that palate based identification methods give better performance than face, signature, heartbeat, voice, and body language with lip motion based methods ${ }^{[10][12][41]}$.

\subsubsection{Limitations of Behavioral Features based Biometrics}

The behavioral features based biometric systems have low accuracy, high FAR, and low FRR. The gait 
signature, lip motion, and body language of a person can be easily mimicked by another professional. The voice of a person changes over time and it may also change in coldness. Few professional experts can match their voice frequency and pitch with other persons. The voice based method is not applicable for identifying a deaf person. Therefore, a single behavioral feature based biometric system will not be sufficient for identifying a person.

\subsection{Why Multimodal Systems are Important?}

Each physiological and behavioral characteristic has its own limitations e.g. lip motion, body language, and voice of a person can be mimicked easily. The physiological features like palm prints, hand geometry, finger veins, and heartbeats have very high false acceptance rate. Thus, a single biometric feature based approach is not reliable and secure. It cannot identify a particular person independently. Therefore, a single feature based biometric system will not be sufficient for fool proof identification of a person. Hence, we need to combine two or more physiological and behavioral characteristics for developing a foolproof multimodal identification system. The multimodal systems have high accuracy and low false rejection rate. The structure of physiological and behavioral characteristics based biometric system is presented in figure 2 .

\section{Fingerprints Based Systems}

This section describes the state-of-the-art of fingerprints based identification methods with advantages, disadvantages, and key features comparison. Traditionally, tokens such as physical key, personal ID cards, and passwords were used to identify a person. The limitations of these automatic tokens are: Tokens can be easily stolen or lost; Password can be guessed or forgotten. Thus, the necessity of a powerful means for identifying a person called biometric personal identification system came into existence ${ }^{[96]}$. A number of finger print based biometric systems have been developed by researchers for identifying an individual. These systems use fingerprint, thumbprint, palm print, finger veins, and hand geometry based features for identity verification ${ }^{[96]}$. The features of palm print image include principle lines, wrinkles, ridges, minutia points, singular points and texture. A low-resolution palm print image with less than $100 \mathrm{dpi}$ (dots per inch) can be used to obtain principle lines and wrinkles. A high-resolution palm print image with at least 400 dpi can be used to obtain minutiae points, ridges and singular points ${ }^{[108]}$.

Fingerprints include the ridge, furrows, minutiae points, orientation of minutiae points, distances between minutiae points, whorl, and curves of fingerprints ${ }^{[39]}$. Hand geometry based identification approaches use the geometric form of hand for confirming the identity of a person. Although, human hands are not unique but, few essential features such as hand length, finger length, finger width, palm length, and palm width may differ from one person to another person. In hand geometry based identification method the hand image is captured using a CCD camera for feature extraction. In image pre-processing stage we extract the hand silhouettes and eliminate artifacts such as guidance pins; user rings; and overlapping cuffs. In hand silhouettes alignment we compute hand length, finger length, finger width, palm length, and palm width. The matching module compares user features with templates stored in database and generates matching score ${ }^{[103]}$.

\subsection{Devices used for Fingerprint Recognition}

Robert Mueller proposed a new approach for identifying a person using low cost equipment. For capturing fingerprints low-cost devises such as Microsoft Lifecam VX300 and Philips SPC630NC are used which manually adjusts sharpness and produces suitable image. The gamma correction is applied to fingerprint images and hybrid algorithms are used to find the minutiae points and skeleton data. For storing data, the mobility token called Micro-SD was used by Mueller which includes a smart card with an ISO compliant ${ }^{[66]}$. Shenglin Yang has given a new idea for identifying a person using fingerprints. It describes a better and efficient embedded fingerprint verification system for the "ThumbPod" embedded device. The ThumbPod is a complete real-time fingerprint recognition module, which includes minutiae extraction and the matching ${ }^{[102]}$.

Chaos and NDFT spread spectrum based technique for fingerprint identification was used by ${ }^{[51]}$. The chaos and NDFT spread spectrum techniques convert templates into audio signals. The templates are encrypted by chaotic encryption and modulated by Chaotic Parameter Modulation (CPM). Template extraction process doesn't require original signals. Because, it is completely blind and it uses Non-uniform Discrete Fourier Transform (NDFT) for data hiding. The data extraction depends on the secrete keys for identifying a particular fingerprint ${ }^{[51]}$. Ailis to Heikki proposed the usage of body weight and body fat percentage with finger print biometrics for personal identification. The method uses optical fingerprint sensor Biometrika FX2000 with FX3 SDK software development kit for identify ing a person [35]. Atsushi Sugiura used Fingerprint User Interface (FUI) for fingerprint recognition. The FUI system identifies the finger when a user touched an input device. It uses pattern matching algorithms of fingerprint identification ${ }^{[84]}$. Aditya Abhyankar has proposed an attractive method which performs following two tasks [1].

Task 1: Use normalized energy and threshold values to check whether a person is alive or not.

Task 2: If the person is alive then take two images of finger prints at the interval of few minutes and use finger print matcher for further identification. 


\subsection{Minutiae Angles and Orientation Fields Based Systems}

Ching-Liang Su developed an accurate and efficient algorith $m$ that automatically calculates and checks the finger-edge and finger-to-finger valleys of hand image ${ }^{[83]}$. In this method the extracted fingerprint geo metries are kept in file. The automatic registration algorithm is used to find the orientations and positions of extracted fingerprints. For image comparison, phase-matching technique is used which includes complex number manipulation. For fingerprint recognition, Structured Query Language (SQL) based searching and manipulation technique using image dilation and interpolation is used ${ }^{[82][83]}$. Gu presented a fingerprint identification system by co mbining global structure and local cues of a fingerprint image ${ }^{[31]}$.

Yang presented finger-veins as a new method for personal identification. First a stable finger-vein region is taken from an imaging sensor and then the fingervein characteristics at different orientations and scales are exploited using Gabor filters. Finger-veins code (FVcode) is constructed with the extracted local and global finger-vein features based on filtered image. Finally, cosine similarity measure classifier and a decision level fusion scheme are adapted for the implementation of finger-vein recognition system ${ }^{[101]}$. The breaking point and bifurcation point of a fingerprint are represented by figure 3 . The breaking points and bifurcation points are called as minutiae points. The angle of orientation of a minutia point is called minutia point orientation field or minutia angle. The minutiae angles of an image are shown in figure 4 [64]

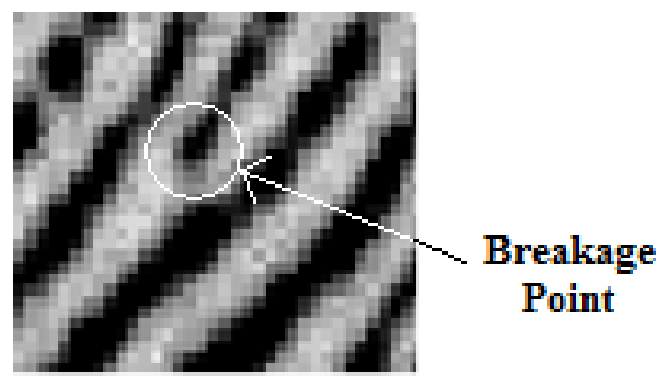

3(a)

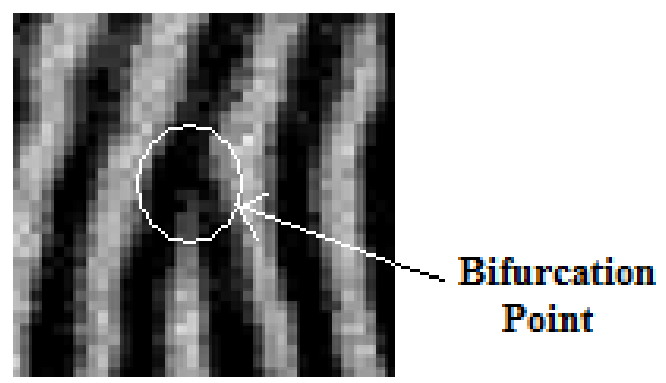

$3(\mathrm{~b})$ Fig.3: Breaking point and bifurcation point of a fingerprint image

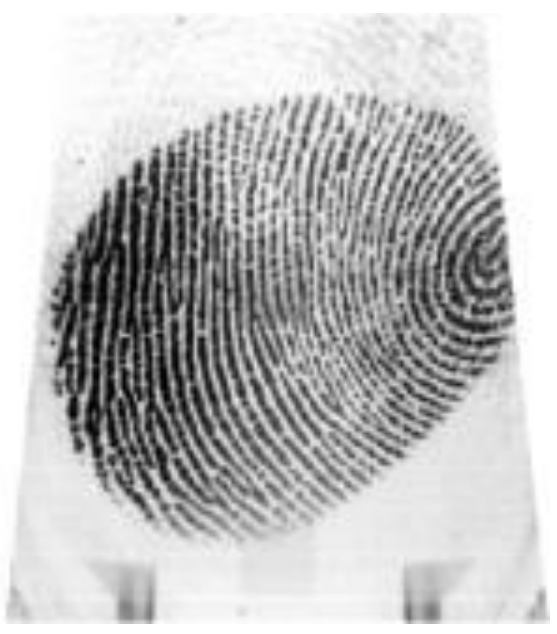

4(a)

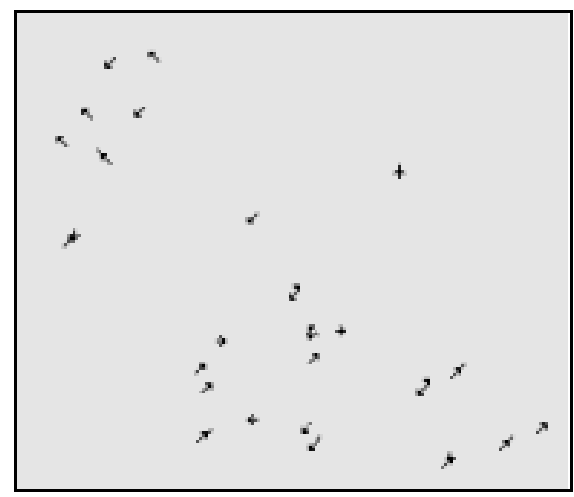

4(b)

Fig. 4: An actual scanned fingerprint image and its minutiae points orientation fields ${ }^{[64]}$.

Danese developed a parallel architecture based BandLimited Phase Only Spatial Correlation (BLPOC) algorithm for implementing matching algorithm. Matching algorith $\mathrm{m}$ is divided into three phases namely: Enrollment, Matching, and Decision. In enrollment phase two fingerprints are enhanced using background elimination and contrast augmentation approaches. The rotation algorithm based on integer pixels is used to rotate the fingerprints at several angles for fingerprints to be compared. For transforming the enhanced fingerprint image Danese used 2-dimensional Discrete Fourier Transform (DFT) ${ }^{[19]}$. Latent fingerprint identification plays an important role in criminal investigations for identifying suspects. Latent fingerprints are inadvertent impressions left by fingers on surfaces of objects. A tremendous progress is seen by researchers in plain and rolled fingerprint matching methods but latent fingerprint matching is still a difficult problem because of poor quality of ridge impressions, small finger area, and large nonlinear distortion. Anil K. Jain proposed a system for matching latent fingerprints with rolled fingerprints enrolled in law enforcement databases. In addition to minutiae matching following others features are matched in this method ${ }^{[44][45]}$ : 
Feature 1: Singularity and ridge quality mapping.

Feature 2: Ridge flow mapping.

Feature 3: Ridge wavelength and skeleton mapping.

The system was tested by matching 258 latent fingerprints in the NIST SD27 database against a background database of 29,257 rolled fingerprints obtained by combining the NIST SD4, SD14, and SD27 databases. The minutiae-based baseline rank-1 identification rate of 34.9 percent was improved to 74 percent when extended features were used ${ }^{[29][68][45]}$.

\subsection{Core, Delta, and Centre Point Distance Based Systems}

Wahab proposed a fingerprint recognition system in which ridge direction based image processing technique was used. The matching technique includes following two steps ${ }^{[94]}$ :

\section{Step 1: Match local features.}

Step 2: Match global features.

Woon Ho Jung established a spiral based fingerprint recognition method. The Woon's algorithm uses following two stages for authentication ${ }^{[49]}$ :

Stage 1: Train ing Stage (Develop a pruned score tree. Compute corelation filters values and score tree values for each node of the tree.)

Stage 2: Verification Stage (verify the matching results).

Woodard used 3-D finger surface features as a biometric identifier for personal identification. Skin folds and crease patterns were extracted from the finger surface as an identifier and a feature template is generated from the curvature based representation of the registered finger images. In image preprocessing four tasks are required: data re-sampling, hand segmentation, finger extraction, and feature template generation. To locate the finger valleys the hull of the contour of hand's silhouette was used ${ }^{[98]}$. The pores matching based fingerprint identification methods used RANSAC (RANdom SAmple Consensus) algorithm for finding the corresponding cores features and finally similarity scores are calculated on the basis of pore matching results ${ }^{[42][106]}$. A ridge pattern flow based data mining approach for fingerprint identification was proposed by ${ }^{[87][9]}$. This approach calculates the numeric code sequence for each ridge flow pattern of the input fingerprint by dividing it into five categories namely Arch, Tented Arch, Left Loop, Right Loop, and Whorl.

The latent-to-rolled/plain matching algorith $\mathrm{m}$ utilizes minutiae, reference points (core, delta, center point of reference), overall image characteristics (ridge quality map, ridge flow map, ridge wavelength map), and skeleton image. Feature extraction algorith $m$ consists of preprocessing and post processing [28] [95]. In feature extraction; reference points, overall image characteristics, and minutiae points are extracted. Ridge validation and minutiae validation algorith ms are used to remove the background noise. Baseline matching algorith $\mathrm{m}$ is used for minutiae matching which includes local minutiae matching, global minutiae matching, and matching score computation ${ }^{[52][68][45]}$.

\subsection{Minutiae Tree and Minutiae Distances based Systems}

Anil K. Jain proposed a fast, robust and simple automatic alignment based fingerprint verification algorith $m$ which uses point pattern matching technique. Minutiae points and minutiae distances are used in this algorithm. The algorithm decomposes minutiae matching into two stages ${ }^{[39][43] \text { : }}$

Stage 1: Alignment Stage (In alignment stage translation, rotation, and scaling between input minutiae are aligned with the template as per specified parameters.)

Stage 2: Matching Stage (In matching stage string matching algorithm is used to match the input minutiae and template minutiae.)

Xinjian Chen proposed Normalized Fuzzy Similarity Measurement (NFSM) algorithm. The template and input fingerprints are aligned and robustness of global alignment is improved using the registration method and local structure matching algorithm ${ }^{[15]}$. The similarities between template and input fingerprints are computed using Normalized Fuzzy Similarity Measure (NFSM). The features considered for similarity match computing are as follow ${ }^{[87][15]}$ :

Feature 1: Number of matched sample points (n).

Feature 2: The mean distance of differences between the matched minutiae pairs (d).

Minutiae based partial fingerprint recognition systems were developed by ${ }^{[46][69]}$. This system may partially identify a person on the basis of few minutiae points of fingerprint. Ravi J. proposed an algorithm for identifying a person using fingerprint minutiae matching score. In this approach Fingerprint Recognition using Minutiae Score Matching (FRMSM) method for matching fingerprints [73]. Fingerprint verification system developed by ${ }^{[37][6]}$ used following two modules:

Module I: Automatic Classification Technique (It is based on minutiae matching algorithms).

Module II: Verification Search Technique (It uses genetic algorithms based searching steps).

Koichi Ito proposed a phase based and feature based fingerprint image matching method. The phase based fingerprint matching used the Phase-only Correlation (POC) and Band Limited Phase Only Correlation (BLPOC) functions. The feature based fingerprint algorithm extracts the corresponding minutiae pairs between the registered image and the input image and it calculates the matching score using BLPOC function ${ }^{[36]}$. Fakourfar proposed a new and effective mechanism 
for fingerprint recognition in maritime environment. Here, database is filled with fingerprints under normal and wrinkled conditions. In wrinkled condition warm water is used for getting clear fingerprint image and then minutiae based fingerprint verification algorithm for score matching is implemented ${ }^{[27]}$. Finger veins pattern based identification is secured and easily verifiable method of personal identification. It uses following steps for identification:

Step I: Perform the finger veins image preprocessing task (Capture the finger veins image by CCD camera).

Step II: Crop the inconvenient image from original image and match the finger veins scores.

The methods for finger print binarization, thinning, and minutiae points extraction using mathematical morphology were presented by ${ }^{[92]}$. For capturing finger print image Classical and U.areU. methods were used. The feature extraction and minutiae detection can be applied after capturing the image using either Classical or UareU. method ${ }^{[92]}$. Figure 5 describes the generalized structure of fingerprints and finger veins.

\subsection{Analyzing Key Features of Fingerprints and Palm Print Based Methods}

The minutiae coordinates, orientation angles, and minutiae distances based methods compare rough and fuzzy set values of a fingerprint or a palm print image whereas cores, deltas, and centre point distance based method compare the patterns and orientations of two images. Minutiae tree based method constructs a minutiae tree for fingerprints and palm print images and this minutiae tree will be compared with the minutiae tree of another online image. Fingerprint based identification methods have low False Acceptance Rate (FAR) and low False Rejection Rate (FRR) values where as palm print, finger veins, and hand geometry based methods have high FAR and FRR values. Therefore, fingerprint based identification methods are preferred over palm print, hand geometry, and finger veins based methods. The acceptability, performance, uniqueness, FAR, and FRR of fingerprints, hand geometry, pal print, and finger veins based methods are presented in table $2^{[104][76][91][15][43][70]}$.

Table 2: Performance measurement of fingerprints based approaches in an automated environment ${ }^{[04][76][91][15][43][70] \text {. }}$

\begin{tabular}{ccccc} 
& Fingerprint & Hand Geometry & Palm Print & Finger Veins \\
\hline Universality & Medium & Medium & High & Medium \\
\hline Uniqueness & High & Medium & Medium & Medium \\
\hline Permanence & High & Medium & Medium & Medium \\
\hline Collectability & Medium & High & Medium & Medium \\
\hline Performance & High & Medium & Medium & Medium \\
\hline Acceptability & Medium & Medium & Medium & Medium \\
\hline Circumvention & High & Medium & Medium & High \\
\hline $\boldsymbol{F A R}$ & Low & Low & High & Medium \\
\hline $\boldsymbol{F R R}$ & Low & Medium & Medium & Low
\end{tabular}

The figures 6 , and 7 represent before-compression and after-compression memory requirement limits of different biometric systems. Here, B1, B2, B3, B4, B5, B6, B7, B8, B9, B10, B11, and B12 represent Hand Writing, Voice, Heartbeat, Hand Veins, Thumbprint, Face Structure, STR of DNA sequence, Palates, Iris Image, Fingerprint, Hand Geometry, Body Language with Lip Motion and Facial Expression respectively. The memory requirement of before-compression is ranging from zero to $1500 \mathrm{~KB}$ and for aftercompression it is ranging from zero to $500 \mathrm{~KB}$. The results of table 2, figure 6 , and figure 7 show that fingerprints based methods give far better matching results in comparison to other methods for online identification ${ }^{[22][41][32][2][16]}$.

\section{Iris Based Identification Systems}

Iris based identification is secure and accurate because, iris image doesn't change for the whole life of a person. The iris image fully develops in the first six months after the birth of a child ${ }^{[11]}$. An Iris based identification system includes following four basis steps for identifying a person ${ }^{[7][11]}$ :

Step 1: Image Acquisition means capturing the iris image using a high resolution camera.

Step 2: Image Preprocessing includes converting the image to a gray scale image and removing noise disturbances.

Step 3: Template matching compares the user templates with templates of database using a matching metric. 
Step 4: Authentication uses the matching metric and declares a person either an authentic person or an

imposter.

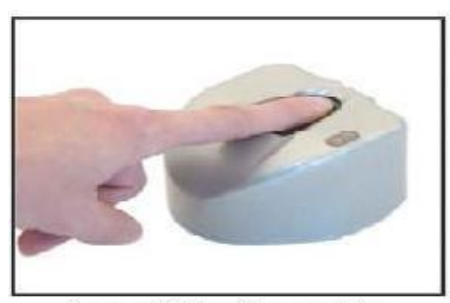

Sensor taking fingerprints

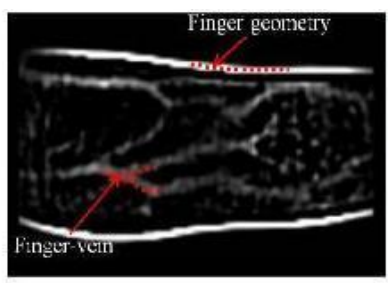

Finger Veins

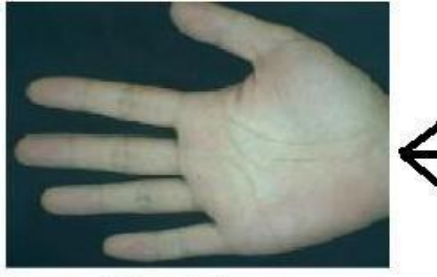

Palmprint

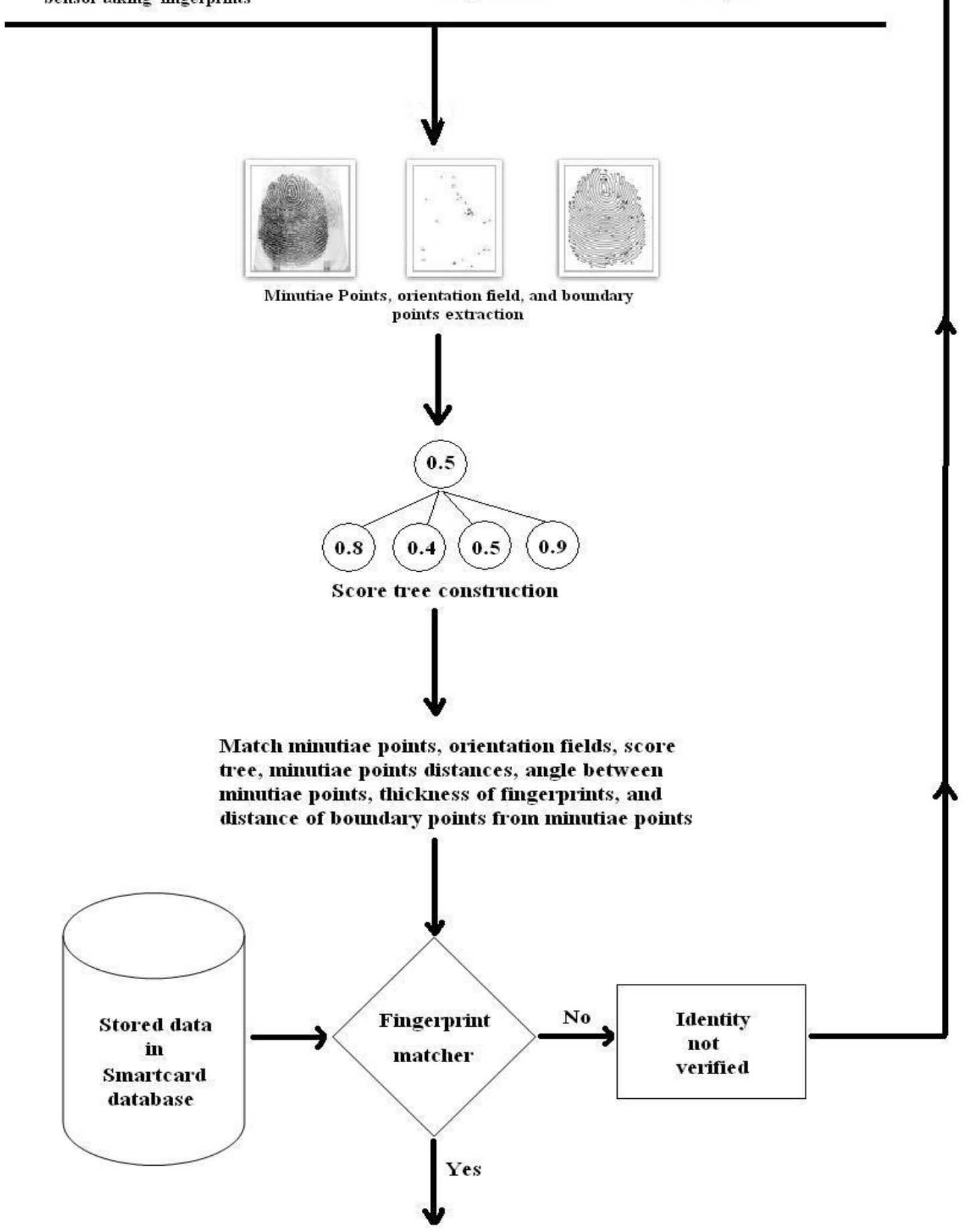

Identity Verified

Fig. 5: Fingerprints and finger veins based automated system structure 


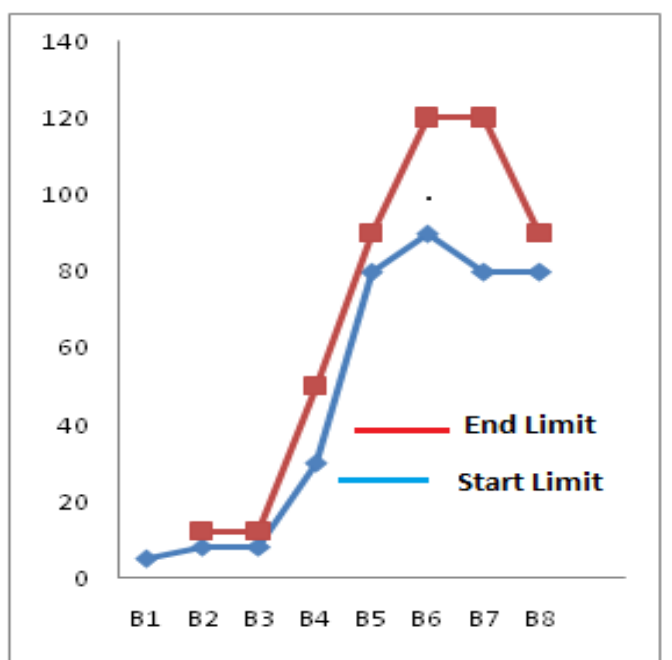

6(a)

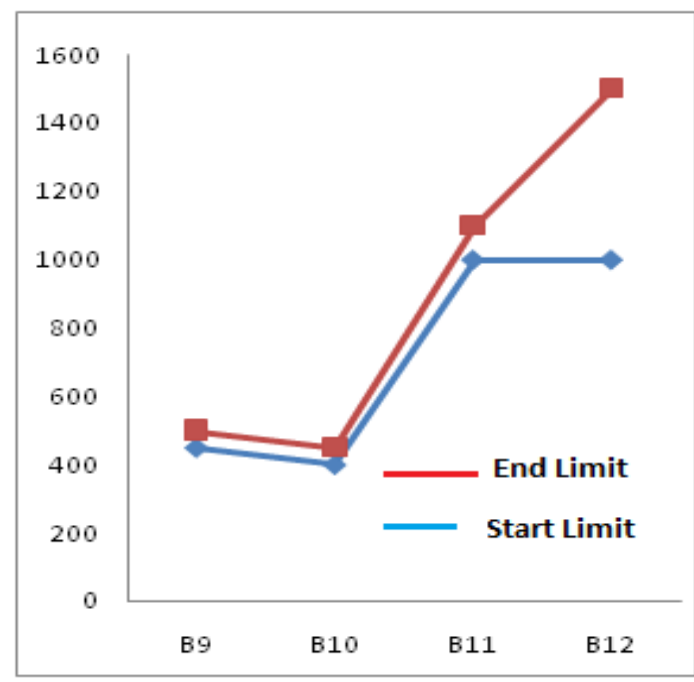

$6(b)$

Fig. 6: Before Compression Memory Requirement Limits of Biometric Systems ${ }^{[104][76][91][15][43][70][22][11][21][47][54][64] \text {. }}$

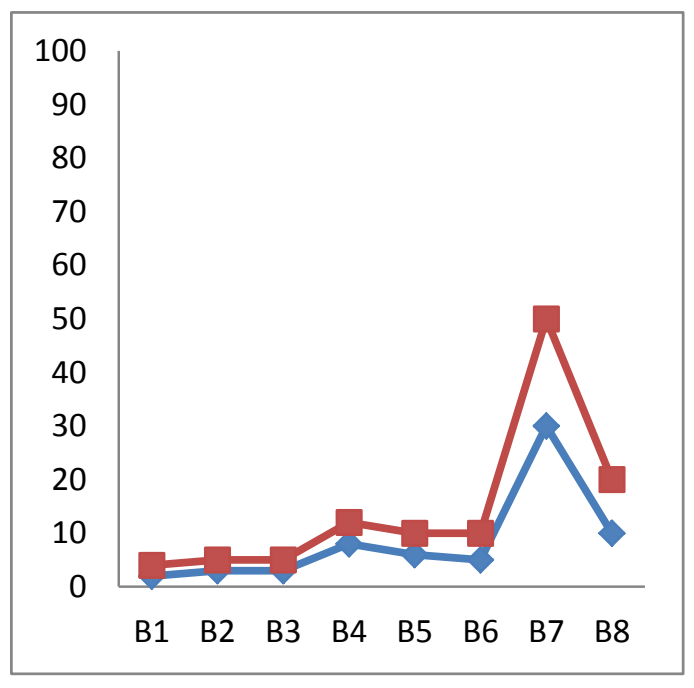

$7(a)$

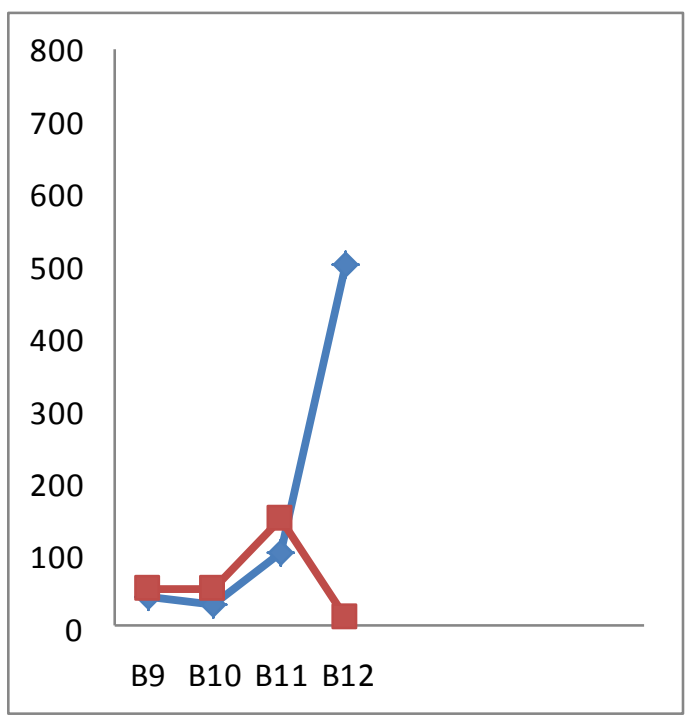

$7(b)$

Fig. 7: After Compression Memory Requirement Limits of Biometric System ${ }^{[104][76][91][15][43][70][22][11][21][47][54][64]}$

Iris based identification methods can be divided into three categories: Wavelet Transformation and Canny Edge Detection, Hamming Distance, Eigen Values and Eigen vector based iris matching methods ${ }^{[7][11]}$.

\subsection{Wavelet Transformation and Canny Edge Detection Based Iris Identification}

Benhammadi developed iris texture analysis based technique for personal identification. The technique uses wavelet packets for binary coding to generating compact signature of iris image datasets ${ }^{[8]}$. The feature extraction and subset selection based techniques for iris identification were used by ${ }^{[4]}$. Here, contour-let transformation technique was used to capture the intrinsic geo metrical structure of iris image and then the iris image was decomposed into a set of directional sub-bands. The texture details were taken from different orientations at various scales. Finally, the system applied SVM (Support Vector Machine) classifiers to identify a person ${ }^{[4]}$. A survey paper based on image understanding for iris biometrics was presented by Bowyer ${ }^{[11]}$. This paper includes Safir, Daugman, and Wilde's approaches. A pattern recognition tool for iris identification which includes edge detection and Hough transformation were suggested by ${ }^{[57[89]}$. It uses Gaber-Filter-Bank for iris localization and pattern matching. An efficient iris recognition algorithm by characterizing key local features was presented by ${ }^{[58]}$. The matching algorithm consists of following steps ${ }^{[58]}$ :

Step I: Construct a set of one dimensional intensity signals for characterizing the important information of original two-dimensional iris image.

Step II: Use a particular class of wavelets to record local sharp variation points in the intensity signals. 
Step III: Match the similarities between sharp variation points of two iris images.

Daugman developed a system which acquires iris image using a LED-based point light source in conjunction with a standard video camera. For matching an iris image, Daugman applied the computation of normalized hamming distances between iris codes ${ }^{[21][22]}$. The Gaussian filter at multiple scales were applied by ${ }^{[97][20]}$ to produce a template and the technique computes the normalized correlation as a similarity measure. Chowhan S. S. presented a survey paper on Biometric System for Security and Identity Management ${ }^{[18]}$. Ramali presented an iris image acquisition and authentication system. The authentication composed of five steps: Histogram; Equalization; Filter; Canny Edge detection; and template matching. This iris acquisition system captures the desired image and compares the stored image with an online image for authentication ${ }^{[72]}$. Serestina Viriri approach detects the largest nonoccluded rectangle part of iris image as a Region Of Interest (ROI) ${ }^{[93]}$. Efficient, robust and fast methods for segmentation of noisy iris images were presented by [105][88]

The Scale Invariant Feature Transform (SIFT) based methods for iris image classification and identification were used by [30][26][47]. The steps of SIFT based methods are as follow:

Step I: Image Acquisition

Step II: Iris Localization

Step III: Determine the darkest point of the image.

Step IV: Find the number of black holes and key points using SIFT.

Step $V$ : Match the key points of the input image with the key points of images in database.

Yong Zhu presented an iris biometric for personal identification. The iris biometric was implemented using Iris Image Acquisition, Image Preprocessing, Feature Extraction, and Iris Identification phases. For texture discrimination wavelet transform was used Yong Zhu. The multi-channel Gabor filter determines the pictorial information in the human cortex. After applying wavelet transformation on an original image we get a set of sub images at different resolution levels. The mean and variance of each wavelet sub-image are extracted as texture features ${ }^{[109]}$.

\subsection{Hamming Distance Based Iris Identification}

James Matey proposed Hamming Distance (HD) and barrel shift based method for comparing two iris images which may differ in resolution, wavelength, occlusion and gaze. A barrel shift is the rotation of cylinder on its axis. Here, circular rotation of iris image is considered as barrel shift about the pupil center. The HD is used to measure the dissimilarities between any two iris images whose two phase code vectors are denoted as $\{$ code $\mathrm{A}$, code $\mathrm{B}\}$ and whose mask bit vectors are $\{$ mask $A$, mask $\mathrm{B}\}$ [59][60]. The HD can be obtained using following mathematical formula:

$$
\begin{aligned}
& H D= \\
& \|(\operatorname{code} A \otimes \operatorname{code} B) \cap(\operatorname{mask} A \cap \operatorname{mask} B)\| \\
& \|(\operatorname{mask} A \cap \operatorname{mask} B)\|
\end{aligned}
$$

Sarasvathi K. proposed a biometric cryptosystem which includes cryptography and biometrics for network security. A cryptographic key is generated from the biometric templates and encrypted minutiae templates of iris are stored in the database ${ }^{[75]}$. John Daugman proposed a new advancement in iris based identification system in which he presented a new method for detecting and modeling inner and outer boundaries with contours ${ }^{[23]}$.

\subsection{Eigen Values Based Iris Identification}

Laplace operator is used for dimensionality reduction of high dimensional data space ${ }^{[7]}$. Belkin and Niyogi used the Eigen values of manifold defined by points in a given featured space. The method is classified as Multi Dimensional Scaling method ${ }^{[7]}$. An efficient eigen values based technique for online iris image compression and identification was presented by ${ }^{[63]}$. The method uses CASIA datasets and completes

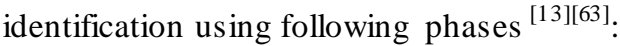

Phase 1: Preprocessing

Phase 2: Estimate Radii and Centre of Iris Ring.

Phase 3: Segmentation (Removing noise disturbances, extracting iris ring, resizing iris image).

Phase 4: Converting Iris Ring into Smaller Matrices.

Phase 5: Calculate Eigen Values.

Phase 6: Compare stored eigen values with online calculated eigen values and display the matching results.

John Daugman proposed a new technique using 2D Fourier Spectrum and 2D Gabor Transform for iris recognition. The method uses following steps to identify an iris image ${ }^{[21][22][23]}$ :

Step 1: Capture at least 70 pixels in iris radius using a monochrome CCD camera.

Step 2: Measure the total high-frequency power in the 2D Fourier spectrum of each frame.

Step 3: Extract the phase modulation using 2D Gabor wavelets.

Step 4: Use the extracted phase for matching and iris recognition.

\subsection{Analysis of Iris Based Biometric Systems}

It is difficult to alter the iris of a person. The iris of a person does not change in his whole life. Iris based 
identification methods are presented in figure 8. The comparis on of False Acceptance Rate (FAR) and False Rejection Rate (FRR) of iris image with thumbprint, finger veins, hand geometry, face, and voice is given in figure 9. The results of figure 9 show that iris based biometric systems have low FAR and medium FRR values. Therefore, Iris based recognition systems are preferred for security measurement of highly secured places but iris based identification methods cannot be used for identifying blind persons. The hamming distance and eigen values based iris identification methods have better robustness and accuracy in comparison to spectrum transformation based iris identification methods.

The FAR and FRR of iris and DNA sequence (discussed in next section) based identification methods measured on different parameters are presented in table 3.

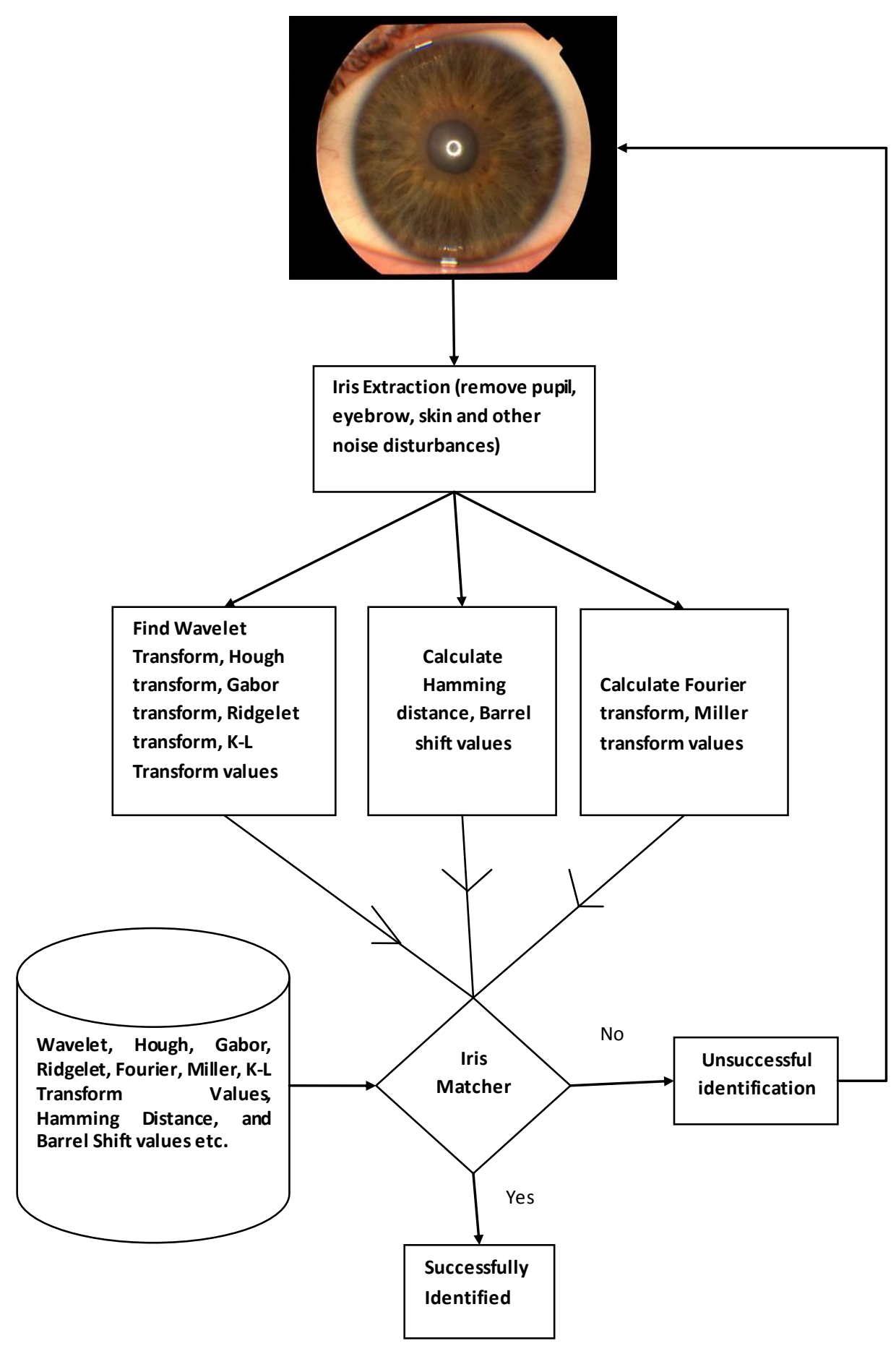

Fig. 8: Iris based identification methods 


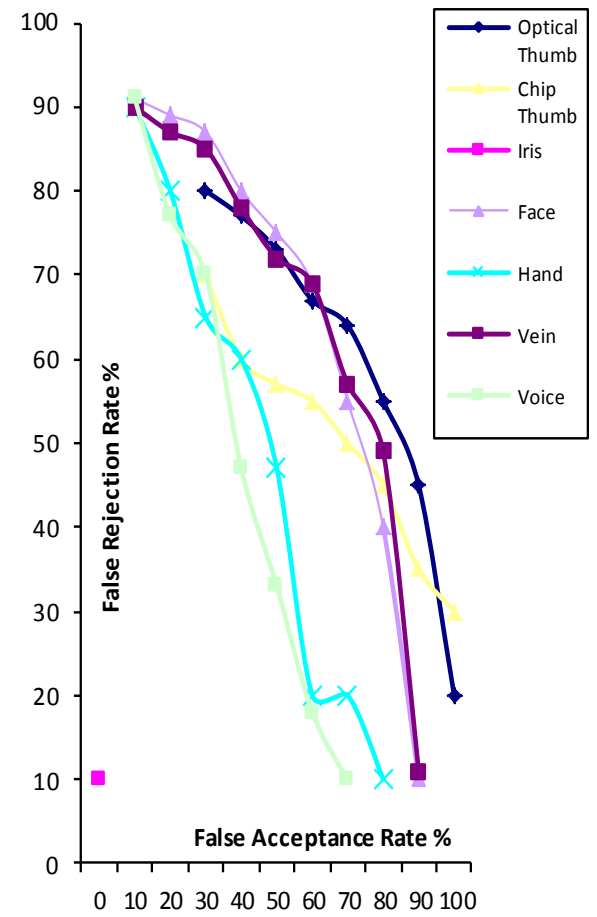

Fig. 9: FAR\% and FRR\% comparison of few important biometric systems ${ }^{[104][76][91][15][43][70][22][11][21][47][54][64]}$.

Table 3: Performance measurement of Iris Image and DNA sequence based identification methods on a soft ware scale ${ }^{[22][11][21][47]}$ $[54][64]$.

\begin{tabular}{ccc}
\cline { 2 - 3 } & Iris & DNA Sequence \\
\hline Universality & High & High \\
\hline Uniqueness & High & High \\
\hline Permanence & High & High \\
\hline Collectability & Medium & High \\
\hline Performance & High & High \\
\hline Acceptability & High & Medium \\
\hline Circumvention & High & Medium \\
\hline $\boldsymbol{F A R}$ & Low & Low \\
\hline $\boldsymbol{F R R}$ & Medium & Low \\
\hline
\end{tabular}

\section{DNA Sequence Based Identification Methods}

Deoxyribonucleic acid (DNA) has a double helix structure and each helix is a linear arrangement of four types of nucleotides: Adenine (A), Cytosine (C), Guan ine (G), Thy mine (T) and unknown nucleotide (N). Here, $\mathrm{N}$ can either $\mathrm{A}$ or $\mathrm{C}$ or $\mathrm{G}$ or $\mathrm{T}$. The DNA sequence can be obtained from a sample of blood, semen, saliva, urine, hair, teeth, bone, tissue, and sweat etc. Every person has a unique DNA sequence excluding identical twins. A sample DNA sequence structure is given in figure $10^{[62][64]}$.

\section{TTTTCGAATTNAACCTCGGTTTNCCTGCCTAACCTC CCAAGT AGCT GGGACT ACAGGCGCCT GCCCGCGCA CCCGGCT AATTTTTAGTAGAGACCGT GTTTCACCG TGTTAGCCAGGATGGT CTCGATCTCCTGAC}

Fig. 10: A sample DNA sequence structure ${ }^{[62][64]}$.

Various algorithms have been proposed to compress the size of DNA sequence data by 1110$][111][85][86][3]$. To compress the DNA sequence we use two main approaches:

Approach I: Statistical Approach [Flocks of fixed length (generally letters) are encoded with respect to their probability of appearance]

Approach II: Substitution Approach [Factors of different length are encoded using a pointer to the previous occurrences of the text].

Hirofumi Tsutsumi presented a new case of personal identification due to detection of rare DNA types from seminal stain. Tsutsumi used a Short Tandem Repeat part of DNA sequence for identifying a person ${ }^{[00]}$. Masaki Hashiyada proposed DNA biometrics based offline identitiy verification. Hashiyada suggested that DNA biometrics doesn't change during the life of a person and therefore it can be used as a reliable source for identifying a living thing. Poly merase chain reaction was used by Masaki for DNA amplification and amplified DNA sequence was matched for identify ing a living thing [33][34]. DNA Compress introduced by ${ }^{[14]}$ uses a two pass strategy is based on substitution (Lempel Ziv style) compression method. In the first pass a specialized programme called Pattern Hunter is used as a preprocessor for finding significant approximate repetitions. The second pass encodes these repetitions by a pointer to their previous occurrences ${ }^{[54]}$

The shape DNA based method was used and deployed by Martin Reuter to identify the shapes of solids and surfaces. Since the spectrum of LaplaceBeltrami operator contains intrins ic shape information. Therefore, it is called "Shape DNA" [74]. Asogawa Minoru analyzed the methods and mechanism for identifying a person using DNA sequence. Minoru used CODIS (Combined DNA Index System) for analyzing DNA sequence ${ }^{[65]}$. A lossless horizontal and vertical method for online DNA sequence compression and identification was presented by ${ }^{[62]}$. In this method the algorithm compresses DNA data first horizontally and then vertically. Here, capital case letters, small case letters, and few special symbols were used to compress and decompress complex DNA sequences. The symbols used by K. N. Mishra for compression and decompression of DNA sequences are as follow ${ }^{[62][64]}$ :

$$
\begin{aligned}
& \mathrm{S}_{\mathrm{A}}=\{\mathrm{B}, \mathrm{D}, \mathrm{E}, \mathrm{F}, \mathrm{H}, \mathrm{I}, \mathrm{J}, \mathrm{K}, \mathrm{L}, \mathrm{M}\} \\
& \mathrm{S}_{\mathrm{C}}=\{\mathrm{O}, \mathrm{P}, \mathrm{Q}, \mathrm{R}, \mathrm{S}, \mathrm{V}, \mathrm{W}, \mathrm{X}, \mathrm{Y}, \mathrm{Z}\} \\
& \mathrm{S}_{\mathrm{G}}=\{\mathrm{a}, \mathrm{b}, \mathrm{c}, \mathrm{d}, \mathrm{e}, \mathrm{f}, \mathrm{g}, \mathrm{h}, \mathrm{i}, \mathrm{j}\} \\
& \mathrm{S}_{\mathrm{T}}=\{\mathrm{k}, \mathrm{l}, \mathrm{m}, \mathrm{n}, \mathrm{o}, \mathrm{p}, \mathrm{q}, \mathrm{r}, \mathrm{s}, \mathrm{t}\} \\
& \mathrm{S}_{\mathrm{N}}=\{\mathrm{u}, \mathrm{v}, \mathrm{w}, \mathrm{x}, \mathrm{y}, \mathrm{z}, \sim, !, @, \%\} \\
& \mathrm{S}=\left\{\mathrm{S}_{\mathrm{A}} \mathrm{U} \mathrm{S}_{\mathrm{C}} \mathrm{U} \mathrm{S}_{\mathrm{G}} \mathrm{US}_{\mathrm{T}} \mathrm{U} \mathrm{S}_{\mathrm{N}}\right\}
\end{aligned}
$$


Set $\mathrm{S}$ is the set of union of all $\mathrm{S}_{\mathrm{X}}$ 's $\left(\mathrm{X} \in \sum\right)$ and it contains all upper case and lowercase letters with few special symbols ${ }^{[64]}$.
The compression and decompression rules used for compressing and decompressing DNA sequences are defined and explained in tables 4 and $5^{[64]}$.

Table 4: DNA Sequence Compression Rules ${ }^{[64]}$

\begin{tabular}{|l|l|l|l|l|}
\hline \multicolumn{1}{|c|}{ S.No. } & \multicolumn{1}{|c|}{ X } & \multicolumn{1}{|c|}{ L $_{\mathbf{x}}$} & \multicolumn{1}{|c|}{ X } \\
\hline R1 & A,C,G,T or $\mathrm{N}$ & $1 \leq \mathrm{L}_{\mathrm{X}} \leq 9$ & $\mathrm{x}=\Psi \mathrm{x}$ & $\mathrm{y}=\mathrm{y} \cdot \mathrm{C}_{\mathrm{X}}$ \\
\hline R2 & A,C,G,T or $\mathrm{N}$ & $10 \leq \mathrm{L}_{\mathrm{X}} \leq 99$ & $\mathrm{x}=\Psi \mathrm{x}$ & $\mathrm{y}=\mathrm{y} \cdot \mathrm{C}_{\mathrm{X}} \cdot \mathrm{Nx}$ \\
\hline
\end{tabular}

Table 5: DNA Sequence Decompression rules ${ }^{[64]}$

\begin{tabular}{|c|c|c|c|}
\hline S.No. & $\mathbf{C}_{\mathrm{L}}, \mathbf{C}_{\mathrm{L}+1}$ & $\mathbf{x}^{\prime}$ & $\mathbf{y}^{\prime}$ \\
\hline $\mathrm{R} 1$ & $\mathrm{C}_{\mathrm{L}} \in \sum$ and $\mathrm{C}_{\mathrm{L}+1} \in \sum$ & $x^{\prime}=x^{\prime}-C_{L}$ & $y^{\prime}=y^{\prime} \cdot S^{c t}$ \\
\hline $\mathrm{R} 2$ & $\mathrm{C}_{\mathrm{L}} \in \sum$ and $\mathrm{C}_{\mathrm{L}+1} \in \partial$ & $x^{\prime}=x^{\prime}-C_{L} \cdot C_{L+1}$ & $\mathrm{y}^{\prime}=\mathrm{y}^{\prime} \cdot \mathrm{S}^{\mathrm{cl1}}$ \\
\hline
\end{tabular}

Ranbir Soram used DNA biometric information for personal identification and described the measurement method of STR data of DNA sequence ${ }^{[81]}$. The generalized structure of methods used for compression and identification of DNA sequence is represented by figure 11 .

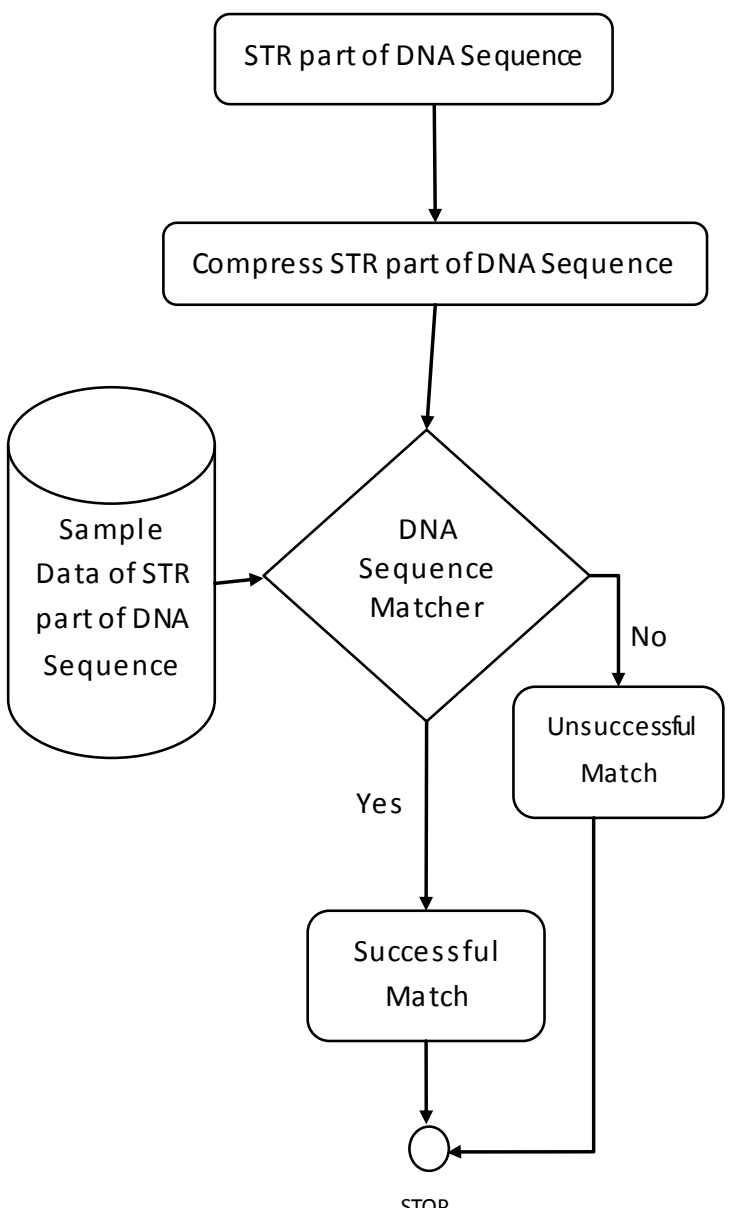

Fig. 11: Structure of compression and matching of DNA sequence

\subsection{Analysis of DNA Sequence Compression and Decompression Methods}

The compression and decompression results of standard DNA datasets for different algorithms are presented in figure 12 and table 6 . The figure 12 compares after-compression-memory-requirements of each algorithm. The results of figure 12 show that DNASC-I gives the best compression result for DNA sequences excluding HUMGHCSA data set. The GeNML method gives the best compression result for HUMGHCSA dataset.

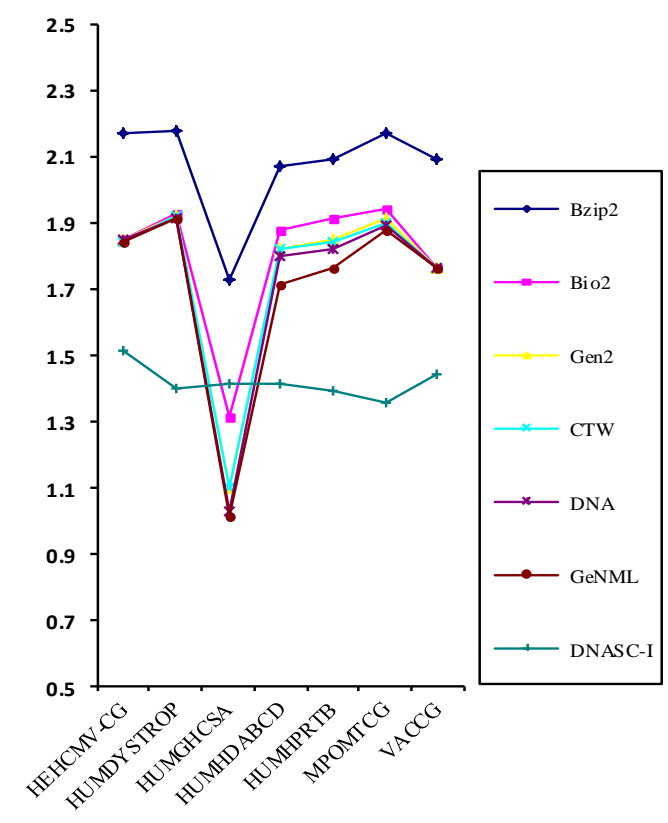
Fig. 12: Graphical Representation of DNA sequence Compression
Methods ${ }^{[64]}$ 
Table 6: Results of compression and decompression of DNA sequences using DNASC-I algorithm ${ }^{[54]}{ }^{[64]}$

\begin{tabular}{|c|c|c|c|c|}
\hline DNA Sequence & $\begin{array}{c}\text { Length Before compression } \\
\text { (in bytes) }\end{array}$ & $\begin{array}{l}\text { Length after Compression } \\
\text { (in bytes) }\end{array}$ & $\begin{array}{l}\text { Compression time } \\
\quad \text { (in seconds) }\end{array}$ & $\begin{array}{l}\text { Decompression Time } \\
\text { (in seconds) }\end{array}$ \\
\hline HEHCMVCG & 229325 & 173132 & 0.6242 & 1.0235 \\
\hline HUMGHCSA & 66495 & 46879 & 0.2254 & 1.1921 \\
\hline MPOMTCG & 686 & 465 & 0.0025 & 0.0182 \\
\hline MTPCAG & 590 & 433 & 0.0054 & 0.0308 \\
\hline VACCG & 1228 & 885 & 0.0045 & 0.0343 \\
\hline HUMDYSTROP & 38770 & 27146 & 0.1306 & 1.0235 \\
\hline HUMHDABC & 58864 & 41650 & 0.1003 & 0.7884 \\
\hline HUMHPRTB & 56737 & 39571 & 0.0989 & 0.6958 \\
\hline
\end{tabular}

Table 6 represents the time required (in seconds) for compression and decompression of standard DNA sequences using DNASC-I algorithm and a processor of $1.40 \mathrm{GHz}{ }^{[64]}$.

The DNA sequence compression and decompression based identification methods cannot be used for online identification of a person because DNA sequence extraction from blood sample, sweat, hair or a cell is an offline process. Since, identical twins share the same DNA sequence. Therefore, DNA sequence based methods cannot be used for identical twins identification.

\section{Multimodal System Based Identification Methods}

A single biometric feature based model is not sufficient for foolproof personal identification. Therefore, we need to combine two or more biometric features for foolproof personal identification. Few of the well-known and established biometric feature combinations are: palate and DNA, heartbeat - head \& voice, iris - DNA \& thumbprint, voice - lip motion \& head movements, Iris - Veins \& hand Geometry, facial expressions - heartbeat \& speech, finger prints - speech $\&$ face $[100][35][25][12][16]$.

A single biometric system may suffer from many problems e.g. sensitivity to noise, intra-class variability, data quality, non-universality (due to incorrect data), intra-class variations (due to incorrect interaction with sensor), inter-class similarities (due to overlap), and spoof attacks [24][53][79]. A multimodal biometric identification system resolves the problems of single biometric system based identification methods. The generalized structure of multimodal bio metric system is represented by figure $13^{[40][81][100][25][24][64]}$.

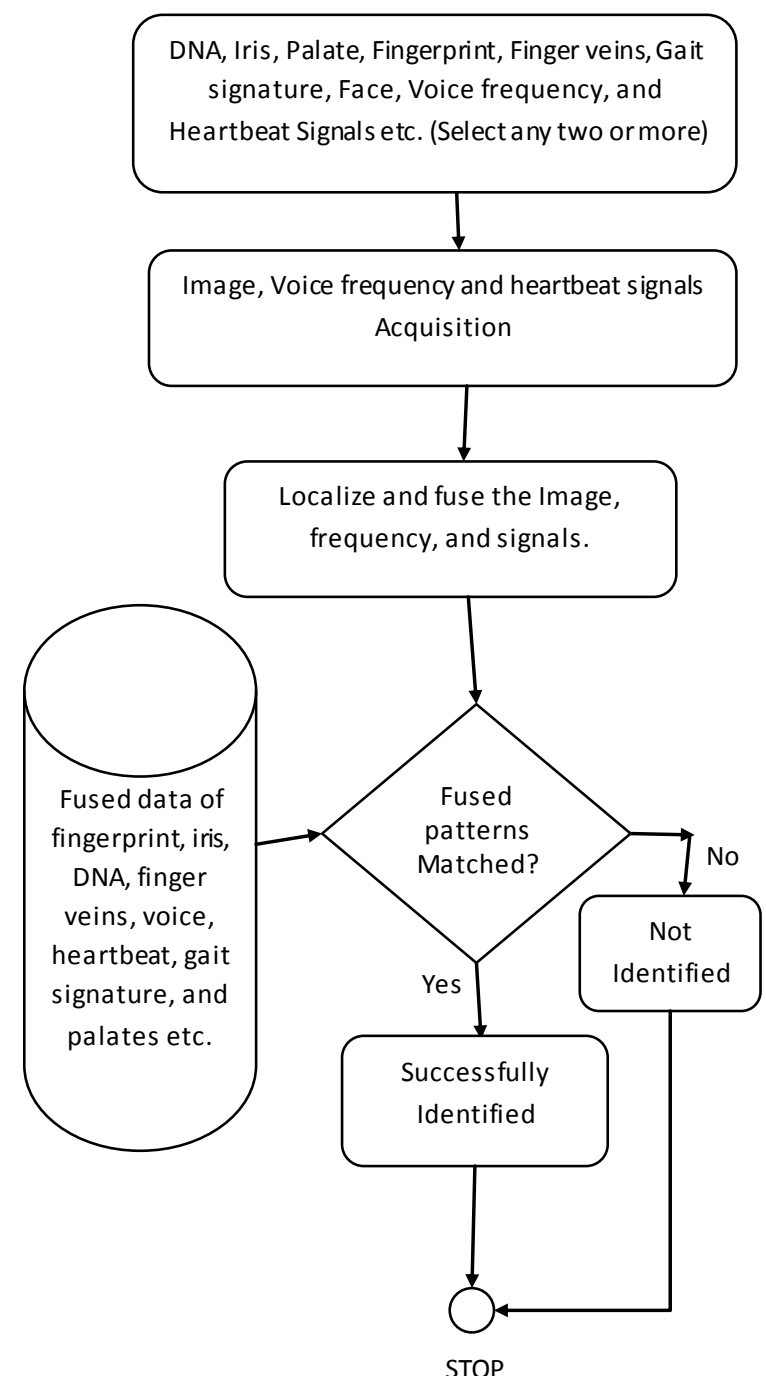

Fig. 13: Personal Identification using Multimodal biometric patterns $[40][81][100][25][24][64]$

In figure 13, the physiological and behavioral features of a person are extracted by using existing techniques and these features are fused together. The 
fused features will be used for further identification of a person including the cases of individuals and identical twins. If the stored multimodal patterns have $80 \%$ above matching with online extracted multimodal patterns then the system will declare a message "Successful Identification" otherwise the system will declare a message "unsuccessful identification".

\subsection{Physiological Features Based Multimodal Systems}

Physiological features based multimodal systems will use the combination of two or more physiological features of a person. Few important comb inations are as follow:

\subsubsection{Palate and DNA Sequence Based Multimodal Systems}

The palate and DNA sequence based multimodal system will be an accurate and foolproof identification system which will be able to identify a living person and a dead person. Every person has unique palatal rugae and DNA sequence excluding the case of identical twins. Identical twins will have different palatal rugae structure but same DNA sequence. If a person losses his body parts (hand, foot, and eye etc.) in an accident then also the combination of palatal rugae and DNA sequence can be used for identity verification [12][64]

\subsubsection{Iris, hand geometry and Finger Veins Based Multimodal Systems}

Iris has low FAR and hand geometry has high FAR where as iris and hand geometry both have medium FRR. Finger veins have low FAR and medium FRR. Therefore, iris-hand geometry-finger veins based multimodal system will have very good acceptability and it will be sufficient to identify a pers on ${ }^{[23][76][101]}$.

\subsubsection{Iris, Thumbprint and DNA Sequence Based Multimodal System}

A new multimodal identification system based on combination of iris, DNA and thumb was proposed by ${ }^{[64]}$. In this system compressed form of STR part of DNA sequence, compressed thumbprint and compressed iris image (Eigen iris) were used. This combination is reliable for identify ing a dead or a living person. The thumb print and iris image are used for identifying a living person. The STR part of DNA sequence can be used for identifying a dead person ${ }^{[64]}$. Iris based systems cannot be used for identifying a blind person. DNA sequence cannot be used for identifying identical twins and thumbprint based sys tem cannot be used for identifying a handicapped (handless) person. Therefore, combination of iris-thumbprintDNA sequence can neutralize the shortcomings of each other and it will be very much useful for identify ing a blind and handicapped identical twin ${ }^{[65][62][64]}$.

A multimodal biometric system for personal identification using iris image and fingerprint fusion was presented by ${ }^{[5]}$. The system used Hamming
Distance (HD) matcher for identifying a person ${ }^{[22]}$. Xue-Zhen-Kuang proposed a weighted cost function based multimodal biometric system. The Xue-ZhenKuang algorith $\mathrm{m}$ is based on local weighted regression, local approximation, and global identification features [100].

\subsection{Behavioral Features Based Multimodal Systems}

The behavioral features include head movement, lip motion, voice, heartbeat, body language, walking style, and gait signature etc. The behavioral features based biometric systems will use valid co mb inations of two or more behavioral features for identify ing a person. Few important behavioral features based multimodal systems are as follows:

\subsubsection{Heartbeat, Head Movement and Voice Based Multimodal System}

The voice frequency of a person can be mimicked and therefore voice based identification system cannot be considered as a foolproof system. If head movement and heartbeats of a person are combined with the voice of that person then it will be a foolproof multimodal system. Because, the voice frequency and head movement of a person can be mimicked by another person but it is not possible by a person to mimic heartbeat-voice frequency-head movement together at the same time. The combination of voice, lip motion and head movement is another valid multimodal system for identifying a person accurately. If two persons talk with each other then both the persons will have unique combination of voice-lip-motion and head movement [25][71].

\subsubsection{Facial Expression, Heartbeat and Speech based Multimodal System}

Every person's heart keeps on pumping until his death. Heart produces signals which are recorded by the Electrocardiograms (ECG). The dig itized heartbeat can be captured and measured using an electronic stethoscope. The digitized heartbeat signals can be stored in the database for matching scores. Combining facial expressions with speech and heartbeat can be a suitable combination for identifying a person because it is difficult to copy facial expression, voice, and heartbeat of a person together at the same time. This combination can be a suitable identification method for verifying the identity of a deaf person ${ }^{[71][16]}$.

\subsection{Combinational Features Based Multimodal Systems}

The combinational features based multimodal systems combine physiological and behavioral features together for identifying a person. Some important combinational features based multimodal systems with their merits and demerits are presented in its subsections. 


\subsubsection{Fingerprints, Facial Expressions and Speech Based Multimodal System}

This combination of multimodal system integrates a face recognition, finger prints verification and speech verification together for identifying a person. Soltane proposed a system which combined the features of face and speech for human identification. Here, Gaussian Mixture Modal (GMM) was used for text independent speech verification. Expectation Maximization (EM) algorithm for maximum likely hood estimation was applied by ${ }^{[80]}$ for personal identification. The combination of Finger print, speech and face based multimodal system is self sufficient to identify a particular person. It is a fact that fingerprint-speechface based multimodal system is being used by law enforcement community ${ }^{[61]}$.

\subsubsection{Head Anatomy and Voice based Multimodal System}

Head structure based identification is the combination of physiological and behavioral elements of human head. In Head Authentication Technique (HAT), the hu man voice and the physiological anato my of the head are used together for identification ${ }^{[10][107]}$. This approach includes capturing and comparing the samples of sounds produced at different locations on the person's head, mouth, and outer-ear. If a person speaks then the resultant resonance is used to generate a biometric absorption template and this biometric template will be used for analyzing the frequencies of data streams. The head authentication technique depends on the way the sound propagates through human head. Sound waves accelerate, decelerate, reflect, refract and attenuate within the complex heterogeneous structure of the head. This method establishes relationship between human voice and the physiological anatomy of the head. The heartbeat can be combined with head anatomy and voice for creating a reliable multimodal system ${ }^{[10][17]}$.

\subsection{Analysis of Multimodal Systems}

A multimodal identification system is reliable and secure method for personal identification. This system uses different combinations of two or more biometric identifiers. If we take a set that includes the combinations of all Multimodal Biometric Systems (MBS) then this set can be represented as $\mathrm{MBS}=\left\{\mathrm{P}_{1}\right.$, $\left.\mathrm{P}_{2}, \mathrm{P}_{3}, \mathrm{P}_{4}, \mathrm{P}_{5}, \quad \ldots \mathrm{P}_{\mathrm{n}}\right\}$ where $\mathrm{P}_{\mathrm{i}}$ will be a multimodal system. Any one of these multimodal systems will be sufficient for foolproof identification of a person.

A Single Multimodal System (SMS) can be represented by following formula:

$$
\text { SMS }={ }^{\mathrm{m}} \mathrm{C}_{\mathrm{n}} \quad \text { where, } 2 \leq \mathrm{n} \leq \mathrm{m}
$$

Here, $m$ represents the total number of biometrics, and $\mathrm{n}$ represents the number of biometrics used for identification.

In figure 14, $\mathrm{X}$-axis represents False Acceptance Rate (FAR) and Y-axis represents Genuine Acceptance
Percentage (GAP). The results of figure 14 show that GAP is very high for fingerprint based methods, low for face based biometric systems and very low for hand geometry based systems. In the figure 15, X-axis represents False Rejection Rate (FRR) and Y-axis represents Genuine Rejection Percentage (GRP) of fingerprint-face-hand geometry based multimodal system. The $\mathrm{X}$-axis values are ranging from 10 to 0.001 in the multiples of $10^{-1}$ and Y-axis values are ranging from 0 to 100 at the difference of 20 . The GRP is very low for fingerprint based methods, high for face based biometric systems and very high for hand geometry based systems ${ }^{[40][20][55][53]}$.

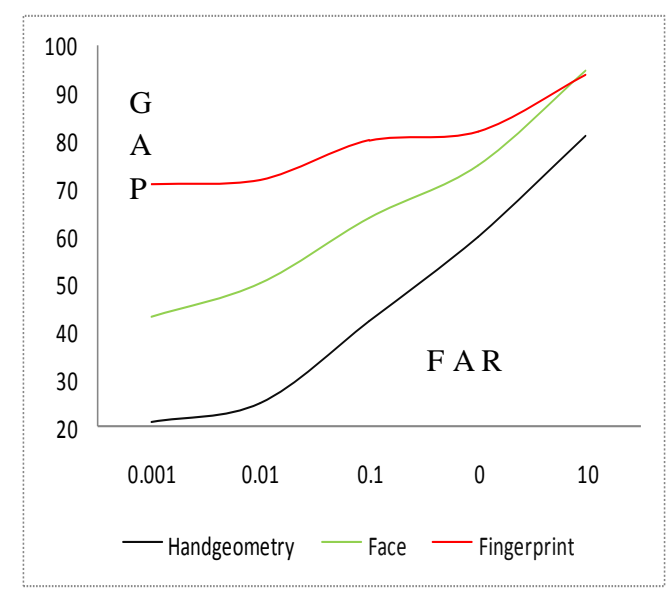

Fig. 14: False Acceptance Rate (FAR) and Genuine Acceptance Percentage (GAP) of Fingerprint, Hand Geometry and Face based multimodal system ${ }^{[40][55][53]}$

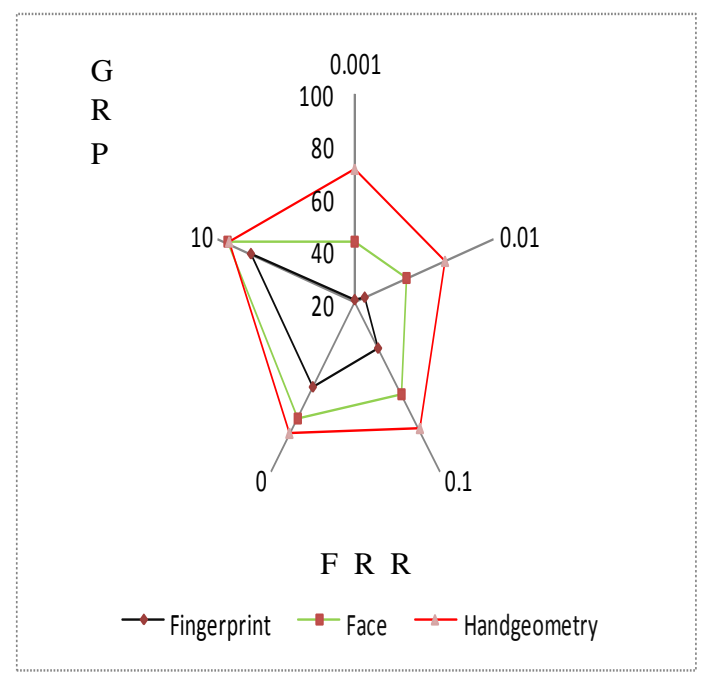

Fig. 15: FRR and GRP of fingerprint, hand geometry, and face based multimodal systems ${ }^{[40][55][53]}$

The FAR and GAP for CASIA (CAS 1, CAS 2, CAS $3)$ datasets are presented in figure 16. The results of figure 16 show that FAR is lower for iris image data sets in comparis on to thumbprints. The iris image has better performance than thumbprint in terms of GAP. The comparis on of FRR and GRP for thumbprint and iris datasets is presented in figure 17. The FRR values 
of figure 17 are varying from 0 to 1 in multiples of $1 / 10^{\mathrm{n}}$ where $\mathrm{n}=0,1,2,3,4$ and GRP values are in the range of 0.90 to 1.0 with an interval of 0.01 [40][55][23][53].

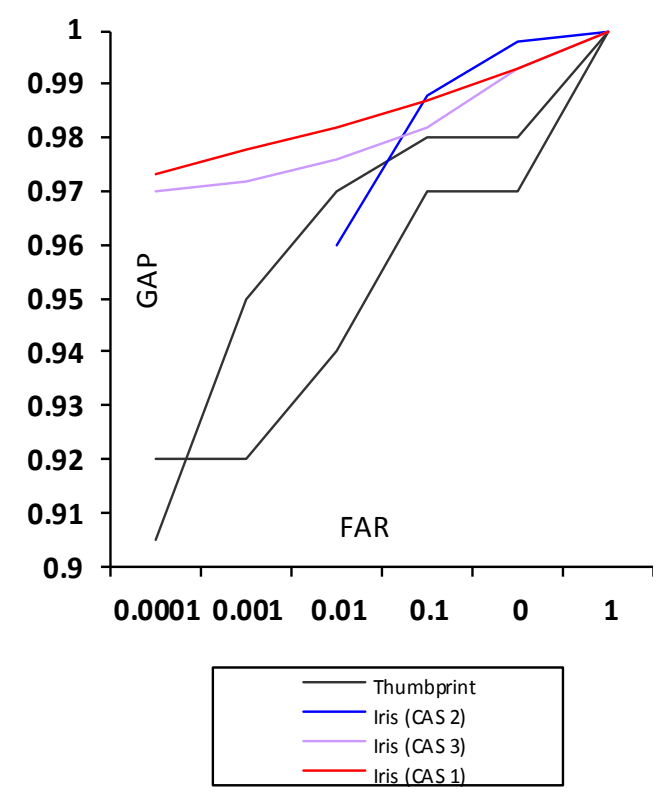

Fig. 16: FAR versus GAP for Thumbprint and Iris based multimodal systems ${ }^{[40][55][23][53]}$
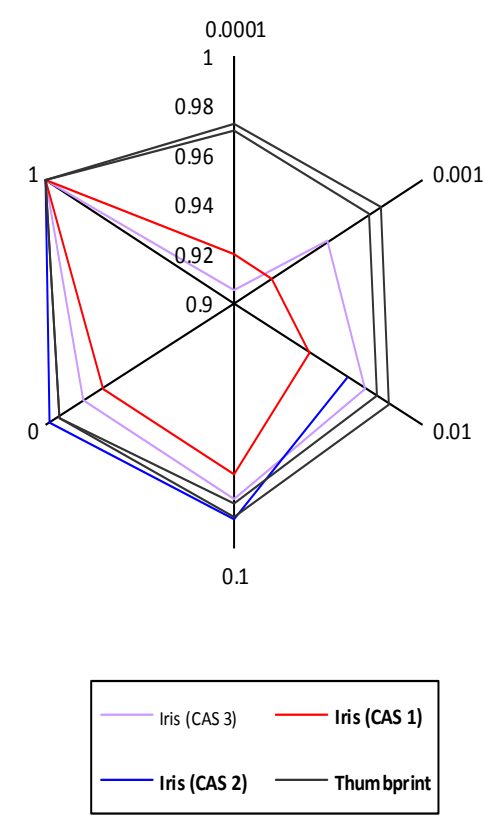

Fig. 17: Comparison of False Rejection Rate (FRR) with Genuine Rejection Percentage (GRP) for thumbprint and iris based multimodal systems ${ }^{[40][55][23][53]}$
Each mu ltimodal system has its own advantages and disadvantages. The heartbeat - head movement \& voice, lip motion - voice \& head movement, and heartbeat speech - facial expressions based multimodal systems have low accuracy and high cost. These systems require a lot of computations for verifying the identity of a person. The palate and DNA based multimodal systems have high accuracy but extracting DNA sequence and palatal rugae patterns will be a time consuming process. The iris - finger veins \& hand geometry based multimodal systems have medium accuracy, medium cost, medium FAR, and medium FRR values. The fingerprints - speech $\&$ face based multimodal systems will require a large amount of time for computations and the system will have low accuracy with high FAR, and high FRR values. The thumbprint - iris \& DNA features based multimodal system has high accuracy, low FAR and low FRR values but the system requires expensive machine setups and high computations.

\section{Conclusion and Future Research Directions}

In this research paper we have discussed the analysis and shortcomings of fingerprints, iris image and DNA sequence based multimodal systems. Bio metric systems based on thumbprint, iris image, finger veins, palates, DNA sequence, voice, and gait signature can identify a person but multiple features based biometric systems give better matching scores in comparis on to single feature based biometric systems. DNA sequence based identification system cannot verify the identity of identical twins and it is not used for online identification.

The multimodal systems e.g. thumbprint-iris-DNA, Palate-Finger veins-fingerprints, head geometry-voicebody language, and heartbeat-voice-body language give better matching results in comparis on to single feature based systems in terms FRR, FAR, GAP, and GRP. A multimodal system based on thumbprint-iris-DNA will be very much useful for identify ing a living person or a dead person. The researchers can further improve the performance existing thumbprint-iris-DNA based multimodal system in terms of accuracy, storage and comparis on time.

In latent fingerprints based identification, the latent fingerprints are collected from crime scene and these are compared with stored fingerprints of database. The researchers can combine latent fingerprint with latent palm print and latent gait signature for foolproof identification of criminals. The researchers need to further improve the performance of latent fingerprints comparison methods and comparis on time [44][45]. Palatal patterns based methods are not mature enough to be used for online personal identification. Therefore, we need to further improve the performance of palatal patterns based online identification methods in terms of FAR, FRR, GAP and GRP ${ }^{[12]}$. The researchers can apply rough set, fuzzy logic, soft computing and neural 
networks based approaches for improving the performance of multimodal systems ${ }^{[77]}$. The ensemble effect of physiological features and the essence of sociological behaviors of individuals and identical twins can be considered as future research directions in this domain.

\section{Acknowledgment}

The researchers would like to thank Professor (Dr.) Ram Pal Singh (Director, Birla Institute of Technology Ranchi, INDIA - Allahabad Campus) for his kind support in completing this research work. The researchers are thankful to Professor (Dr.) Ajay Chakrabarty (Vice Chancellor, Birla Institute of Technology, Mesra, Ranchi, India) for creating academic and research oriented environment at the institute.

The research team would like to thank Dr. R. Sukesh Kumar (Professor \& Head, Dept. of Computer Sc. \& Engg., B.I.T. Mesra, Ranchi) and Dr. G. Sahu (Professor \& Head, Dept. of I.T., B.I.T. Mesra) for encouraging us to write quality research papers.

\section{References}

[1] Abhyankar Aditya, Schuckers Stepanie. Integrating a wavlet based perspiration liveness check with fingerprint recognition. Pattern Recognition, Elsevier, 2009, 42: 452-464.

[2] Anthonioz A., Eg li N., Champod C., Neu mann C., et al. Level 3 Details and their Role in Fingerprint Identification: A Survey among Practitioners. Journal of Forensic Identification, 2008, 58(5): 562-589.

[3] Apostolico Alberto, Lonardo Stefano. Compression of Biological Sequences By Greedy Off-Line Textual Substitution. In: Data Compression Conference, 2000, 143 - 150.

[4] Azizi Amir, Pourreza Hamid Reza. Efficient Iris Recognition Through Improvement of Feature Extraction and subset Selection. International journal of computer science and information security, 2009, 2(1): $71-80$.

[5] Baig Asim, Ahmed Bouridane, Fatih Kurugollu et al. Fingerprint - Iris fusion based identification system using a single Hamming Distance Matcher. International journal of Bio- science and BioTechnology, 2009,1(1): 9 - 12.

[6] Bana Sangram, Kaur Davinder. Fingerprint Recognition using Image Seg mentation. Journal of Advanced Engineering Science and Technology, 2011, 5(1): $12-23$.

[7] Belkin M., Niyogi P. Laplace Eigen maps for Dimensionality Reduction and Data
Representation. Neural Computation, MIT Press, 2003, 15: 1373-1396.

[8] Benhammadi Farid, Kihal Nassima. Personal Authentication Based on Iris Texture Analysis. In: The 6th ACS/IEEE International Conference on Computer Systems and Applications, AICCSA, 2008, $537-543$.

[9] Bhuyan Hussain Monowar, Saharia Sarat, Bhattacharyya K. An Effetcive Method for fingerprint Classification. International Arab Journal of e-Technology, 2010, 1(3): 89 - 97.

[10] Bohm Igor, Tester Florian. Biometric Systems: Report. Department of Telecooperation. University of Linz, Austria, 2004, 1-7.

[11] Bowyer Kevin W., Hollingsworth Karen, Flynn Partrick J. Image understanding for iris biometrics : A survey. Computer Vision and Image Understanding, Elsevier,2008, 110: 281-307.

[12] Caldas I. M., Magathaes T., Afonso A. Establishing Identity Using Cheiloscopy and palatoscopy. Forensic Science International, 2007, 165: $1-9$.

[13] CASIA. Iris Image Database-Iris Recognition Research Group of National Laboratory of Pattern Recognition (NLPR), Institute of Automation. Chinese Academy of Sciences, July 2010, [Online], Available: http://www.sinobiometrics.com

[14] Chen Xin, Li Ming, Ma Bin et al. DNA Compress: Fast and Effective DNA Compression. Bioinformatics Applications Note, 2002, 18(12): $1696-1698$.

[15] Chen Xinjian, Tian Jie, Yang Xin. A New Algorithm for Distorted Fingerprints Matching Based on Normalized Fuzzy Similarity Measure. IEEE Transactions on Image Processing, 2006, 15(3): 767-776.

[16] Chetty G., Wagner M. Robust Face-Voice Based Speaker Identity Verification Using Multilevel Fusion. Image and Vision Computing, Elsevier, 2009, 26: 1249-1260.

[17] Choras Michal. Ear Biometrics Based on Geo metrical Feature Extraction. Electronic Letters on Computer Vision and Image Analysis, 2005, 5(3), 84-95.

[18] Chowhan S. S., Shinde G. N. Iris Biometrics Recognition Application in Security Management. ICCES, 2008, 6(1):1-12.

[19] Danese G., Giachero M., Leporati F. et al. An embedded multi-core Biometric identification system. Microprocessors and Microsystems, Elsevier, 2011, 35(5): 1-12.

[20] Daugman John. Demodulation by complex-valued wavelets for stochastic pattern recognition. 
International Journal of Wavelets, Multiresolution and Information Processing, World Scientific, 2003, 1(1): 1-17.

[21] Daugman John. How Iris Recognition works. IEEE Transaction on Circuits and Systems for Video Technology, 2004, 14(1): 21-30.

[22] Daugman John. Probing the uniqueness and randomness of Iris Codes: Results from 200 billion iris pair comparisons. In: Proceedings of the IEEE, 2006, 94(11): 1927-1935.

[23] Daugman John. New methods in iris recognition. IEEE Trans. on Systems, Man, Cybernetics B, 2007, 37(5): 1167-1175.

[24] Doroteo T. Toledano Usability evaluation of multi-modal biometric verification systems. Interacting with Computers, Elsevier, 2006, 18: 1101-1122.

[25] Etignu H., Erzin E., Yemez Y. et al. Multimodal Speaker/Speech Recognition Using Lip Motion Lip Texture and Audio. Signal Processing, Elsevier, 2006, 86(12): 3549-3558.

[26] Fakhry Hussein H., Cardozo Benedict Bernard. Research and Development of Iris Based Recognition System For Identification and Secure Authentication, IT Emerging Commercial Capabilities and Terroris m. ProCon Ltd. Bulgaria, 2006, $39-57$.

[27] Fakourfar Hourieh, Belongie Serge. Fingerprint Recognition System Performance in the maritime Environment. In: Workshop on Applications of Computer Vision, Snowbird, 2009, 1- 5.

[28] [28] Feng J., Ouyang Z., Cai A. Fingerprint Matching Using Ridges. Pattern Recognition, 2006, 39(11): 2131-2140.

[29] [29] Feng J. Combining Minutiae Descriptors for Fingerprint Matching. Pattern Recognition, 2008, 41(1): 342-352.

[30] Ganeshan Balaji, Theckedath Dhananjay, Young Rupert et al. Biometric iris recognitions system using a fast and robust localization and alignment procedure. Optics and Lasers in Engineering, 2006, 44: 1-24.

[31] Gu J., Zhu J., Yang C. Fingerprint Recognition by Combining Global Structure and Local Cues. IEEE Trans. on Image Processing, 2006, 15(7): 1952-1964.

[32] Hao Feng, Daugman John, Piotr Zielinski. A fast search algorithm for a large fuzzy database. IEEE transaction on information forensics and security, 2008, 3(2): $203-212$.

[33] Hashiyada Masaki. DNA Biometrics. InTech publisher Japan, 2004, 139-154.
[34] Hashiyada Masaki. Development of biometric DNA ink for Authentication Security. Tohoky Journal of Exp. Med., 2004, 204(2): 109-117.

[35] Heikki Ailisto, Elena Vildjiounaite, Mikko Lindholm et al. Soft Biometrics Combining Body Weight and Fat Measurements with Fingerprint Biometrics. Pattern Recognition Letters, Elsevier, 2006, 27(5): 325-334.

[36] Ito Koichi, Morita Ayumi, Aoki Takafumi et al. A Fingerprint Recognition Algorithm Combining Phase-Based Image Matching and Feature-Based Matching. Lecture Notes in Computer Science, ICB2006, 316-325.

[37] Jaam Jihad, Rebaiaia Mohamed, Hansah Ahamad. A Fingerprint Minutiae Recognition System Based on Genetic Algorithms. International Arab Journal of Information Technology, 2006, 3(3): $242-248$.

[38] Jain Anil. K., Hong Lin, Bolle Ruud. On-Line Fingerprint Verification. IEEE Transactions on Pattern Analysis and Machine Intelligence, 1997, 19(4): 302-314.

[39] Jain Anil K., Hong Lin, Pankati Sharath et al. An Identity-Authentication System Using Fingerprints IEEE Transactions on Pattern Analysis and Machine Intelligence, 1997, 85(9): 1365-1388.

[40] Jain Anil K., Hong Lin, Kulkarni Vatin. A Multimodal Biometric System Using Fingerprint, Face and Speech. IEEE Transactions on Patterm Analysis and Machine Intelligence, 1998, 20(12): 1295 - 1307.

[41] Jain Anil K., Chen Y., Demirkus M. Pores and Ridges: High- Resolution Fingerprint Matching Using Level 3 Features. IEEE Trans. Pattern Analysis and Machine Intelligence, 2007, 29(1): $15-27$.

[42] Jain Anil K. Evaluation of Latent Fingerprint Technologies. 2007, [Online], Available: http://fingerprint.nist.gov/latent/elft07/

[43] Jain Anil K., Feng J., Nagar A. et al. On Matching Latent Fingerprints. In: Proc. of IEEE Computer Vision and Pattern Recognition Workshop on Biometrics, 2008, 1-8.

[44] Jain Anil K., Feng Jianjiang. Latent Palm Print Matching. IEEE Transactions on Pattern Analysis and Machine Intelligence, 2009, 31(6): 1032 1047.

[45] Jain Anil K., Feng Jiang. Latent Fingerprint Matching. IEEE transactions on Pattern Analysis And Machine Intelligence, 2011, 33(1): 88-100.

[46] Jea T. Y., Govindaraju V. A Minutia-Based Partial Fingerprint Recognition System. Pattern Recognition, Elsevier, 2005, 38(10): 1672-1684. 
[47] Jhamb Mansi, Khera Vinod K. Iris based human recognition system. International Journal of Biometrics and Bioinformatics, 2011, 5(1): 1 - 9 .

[48] John Vacca. Biometric Technologies and Verification Systems. A book of ButterworthHeinemann publisher, Elsevier, 2007, 1 - 625.

[49] Jung Woon Ho. Fast fingerprint Recognition using Spiral. Department of Electrical and Computer Engineering, Carnegie Mellon University, Pittsburgh, 2011, 1-7, [online]. Available: http://www.ece.c mu .edu/ pueschel/teaching/18799B-CMU-spring05/material/woonho.pdf

[50] Kapali S, Townsend G., Richards L. et al. Palatal Rugae Patterns. Australian Aborigines and Caucasians. Australian Dental Journal, 1997, 42: 129-133.

[51] Khan M. K., Xie Ling, Zhang Jiashu. Choas and NDFT based spread spectrum concealing of fingerprint-biometric data into audio signals. Digitla Signal Processing, Academic Press, 2010, 20(1): 179-190.

[52] Komarinski P. Automated Fingerprint Identification Systems (AFIS). A book published by Academic Press, Elsevier, 2005, 1 - 249.

[53] Ko T., Krishnan R. Fingerprint and Face Identification for Large User Population. Systematics Cybernetics and Informatics, 1(3): 87-92.

[54] Korodi Gregely, Tabus Ioan. An Efficient Normal Maximum Likelihood Algorithm for DNA sequence compression. ACM Transactions on Information Systems, 2005, 23(1): $3-34$.

[55] Ku mar A jay, David C.M., Wong B. Et al. Personal authentication using hand images. Pattern Recognition Letters, Elsevier, 2006, 27(13): 1478 1486.

[56] Kumar V.D. A mbeth, Ramkrishnan M. Footprint Recognition using Modified sequential Haar Energy Transform (HSHET). International Journal of Computer Science Issues, 2010, 7(5): 47-51.

[57] Ma L., Yunhong, W., Tieniu, T. Iris Recognition Using Circular Sy mmetric Filters. In: Proceedings of International Conference on Pattem Recognition, 2002, 2: 414-417.

[58] Ma L., Tieniu T., Yunhong W. et al. Efficient Iris Recognition by Characterizing Key Local Variations. IEEE Transactions on Image Processing, 2004, 13(6): 739 - 750.

[59] Matey James R., Hanna K., Kolcyznski R. et al. Iris on the move: Acquisition of images for iris recognition in less constrained Environments. Proc. IEEE, 2006, 94(11): 1936 -1947.
[60] Matey James R., Broussard Randy, Kennell Lauren. Iris image segmentation and sub-optimal images. Image and Vision Computing,Elsevier, 2010, 28(2): 215-222.

[61] Micheloni Christian, Sergio Canazza, GianLucaForesti. Audio Video Biometric Recognition for Non-collaborative Access Granting. Journal of Visual Languages and Computing, Elsevier, 2009, 20: 353-367.

[62] Mishra K. N., Agrawal A., Srivastava P.C., et al. An Efficient Horizontal and Vertical Method for Online DNA Sequence Compression. International Journal of Computer Applications, 2010, 3(1): 39-46.

[63] Mishra K. N., Agrawal A., Srivastava P. C., et al. An Efficient Eigen Values Based Technique for Online Iris Image Compression and Identification. International Journal of Information Acquisition, World Scientific, 2011, 8(2): 133-152.

[64] Mishra K. N., Srivastava P. C., Agrawal A. et al. A Framework towards Using Eigen Iris, Minutia Thumb and DNA Sequence Features for Personal Identification. International Journal of Information Acquisition, World Scientific, 2011, 8(3): 197-225.

[65] Minoru Asogawa. DNA-based personal identification and its technologies. Nec Technical Journal, 2010, 5(3): 34-38.

[66] Mueller Robert, Sanchez-Reillo Raul. An Approach to Biometric Identity Management Using Low-Cost Equipment. In: Proceedings of IEEE Conference on IIH-MSP, 2009, 1096 - 1100.

[67] Nicolae Duta. A Survey of Biometric Technology Based on Hand Shape. Pattern Recognition, Elsevier, 2009, 42(11): 2797 - 2806.

[68] "NIST Mated Fingerprint Card Pairs 2 (MFCP2)," NIST Special Database 14, 2010, [Online], Available: http://www.nist.gov/srd/nistsd14.htm

[69] Parra Philippe. Fingerprint minutiae extraction and matching for identification procedure. Department of CS\&E, University of California, 2011, $1-8$, [Online], Avaliable: http://cseweb.ucsd.edu/classes/wi07/cse190a/reports/pparra.pdf

[70] Pathak Preeti. Image Compression Algorithms for Fingerprint System. International Journal of Computer Science Issues, 2010, 7(3): 45 - 50.

[71] Phua Koksoon, Chen Jianfen, Dat Tran Huy et al. Heart sound as a biometric. Pattern Recognition, Elsevier, 2008, 41(3): 906 - 919.

[72] [72] Ramali Mohamad, Akmar Nurul Kamrudin, Saufi Muhammad et al. Iris Recognition for personal Identification. In: International 
Conference on Electrical Engineering, Okinawa, Japan, No. 0-099, 2008, 1 - 5.

[73] Ravi J, Raja K. B., Venugopal.K.R Fingerprint Recognition using minutia score matching. International Journal of Engineering Science \& Technology, 2009, 1(2): 35-42.

[74] Reuter Martin, Wolter Franz-Erich, Peinecke Niklas. Laplace-Beltrami Spectra as Shape DNA of surface and solids. Computer Aided Design(CAD), Elsevier, 2009, 41(10): 739 - 755.

[75] Sarasvati K., Jayram B., Balasubramanian R. Iris Biometrics Based Authentication and Key Exchange System. International Journal of Engineering and Technology, 2011, 3(1): 102-108.

[76] Septimiu Crisan, Ioan Gavril T. et al. Radiation optimization and image processing algorithms in the identification of hand vein patterns. Computer Standards and Interfaces, Elsevier, 2010, 32: 132 $-140$.

[77] Shen Qiang, Jensen Richard. Rough Sets Their Extensions and Applications. International Journal of Automation and Computing, Springer, 2007, 4(3): $217-228$.

[78] Shetty Mahabalesh, Premalatha K. Study of Palatal Rugae Pattern Among the Student Population in Mangalore. Journal of Indian Academy of Forensic Medicine, 2011, 33(2): 112 115.

[79] Singh Mini, Chabra Sumit. A Survey of Multimodal Biometrics. International Journal of Computer Sc. \& its Applications, 2011, 157 - 160. [Online], Available: http://s eekdlib.uacee.org/files/a375.pdf

[80] Soltane Mohamed, Doghmane Noureddine, Guersi Noureddine. Face and Speech Based Multi-Modal Biometric Authentication. International Journal of Advanced Science and Technology, 2010, 21: $41-$ 56.

[81] Soram Ranbir, Khomdram Memeta. Biometric DNA and ECDLP- based personal Authentication System: A Superior Posse of Security. International Journal of Computer Science and Network Security, 2010, 10(1): 1-9.

[82] Su Ching-Liang. Finger extraction, finger image automatic registration, and finger identification by image phase matching. Applied Mathematics and Computation, 2007, 188(1): 912-923.

[83] Su Ching-Liang. Hand image recognition by the techniques of hand shape scaling and image weight scaling. Expert Systems with Applications, Elsevier, 2008, 34(4): 2976 - 2987.

[84] Sugiura Atsushi, Koseki Yoshiyukin. A user Interface Using Fingerprint Recognition Holding Commands and Data Objects on Fingers. In:
Proceedings of the 11th annual ACM symposium on UIST, 1998, $71-79$.

[85] Tahi Fariza, Grumbach Stephen. A New Challenge for Compression Algorithms: Genetic Sequence. Information Processing and Management, Elsevier, 1994, 30(6): $875-886$.

[86] Tahi Fariza, Grumbach Stephen. Compression of DNA Sequences: Extended Abstract, 1994, 1 - 11.

[87] Tico M., Kuosmanen P. Fingerprint Matching Using an Orientation-Based Minutia Descriptor. IEEE Trans. on Pattern Analysis and Machine Intelligence, 2003, 25(8): 1009-1014.

[88] Tieniu T., Zhaofeng H., Zhenan H. Efficient and Robust Segmentation of Noisy Iris Images for Non-cooperative Iris Recognition, Image and Vision Computing, Elsevier, 2010, 28: 223 - 230.

[89] Tisse C. L. Person Identification Technique Using Human Iris Recognition. Journal of System Research, 2003, 4: 67-75.

[90] Tsutsumi Hirofumi, Komuro Toshinobu, Rei Mukoyama et al. A case of personal identification due to detection of rare DNA types from seminal stain. Journal of Oral Science ,2009, 51(4): 645650 .

[91] Vajna Z. K. A Fingerprint Verification System Based on Triangular Matching and Dynamic Time Warping. IEEE Trans. Pattern Analysis and Machine Intelligence, 2000, 22(11): 1266- 1276.

[92] Virginia Espinosa-Duro. Minutiae Detection Algorithm for Fingerprint Recognition. In: IEEE AESS Systems Magazine, 2002, 7-10.

[93] Viriri Serestina, Ju les R Tapamo. Improving irisbased personal identification using maximum recatanguler region detection. In: International Conference on Digital Image Processing, IEEE computer society, 2009, 421 - 425.

[94] Wahab A., Chin S. H., Tan E.C. Novel approach to automated fingerprint recognition. IEEE Proceedings on Vision Image Signal Process, 1998, 145(3): 160-166.

[95] Wan D., Zhou J. Fingerprint Recognition Using Model-Based Density Map. IEEE Trans. on Image Processing, 2006, 15(6): 1690-1696.

[96] Wang P.S.P., Yanushkevich S.N. Biometric technologies and applications. In: Proc. of IASTED AIA (Artificial Intelligence Applications), Innsbruck, Austria, 2007, 226-231.

[97] Wildes R. P. Iris Recognition: An Emerging Biometric Technology. Proceedings of IEEE, 1997, 85(9): $1348-1363$.

[98] Woodard Damon L., Flynn Patrick J. Finger Surface as biometric identifier. Computer Vision 
and Image Understanding, Elsevier, 2005, 100(3): 357-384.

[99] [Online], Available: http://www.biometrics.gov/documents/biohistory. pdf , November 2011.

[100] Xue Zhen-Kuang, Li Shao-Yuan A multimodal identification algorithm based on weighted cost function and application in thermal process. Acta Automatica Sinica Journal, 2005, 31(3): $470-474$.

[101]Yang Jinfeng, Shi Yihua, Yang Jinli. Personal identification based on finger-vein features. Computers in Human Behavior, Elsevier, 2011, 27(5): $1565-1570$.

[102] Yang Shengling, Verbauwhede Ingrid. A Real time, Memeory Efficient Fingerprint Verification system. In: Proc. of IEEE International Conference on Acoustics, Speech and Signal Processing, 2004, 5: 189 - 192.

[103] Yoruk Erdem, Dutagaci, Helin, Sankur Bulent. Hand biometrics. Image and Vision Computing, Elsevier, 2006, 24(5): 483 - 487.

[104] Zhang David, Wai Kin Kong. Online Palm Print Identification. IEEE Transaction on Pattern Analysis and Machine Intelligence, 2003, 25(9): 1041-1050.

[105] Zhaofeng H., Tieniu T., Zhenan S. et al. Toward Accurate and Fast Iris Segmentation for Iris Biometrics. IEEE Transactions on Pattern Analysis and Machine Intelligence, 2009, 31(9): $1670-1684$.

[106] Zhao Qijun, Zhang Lei, Zhang David et al. Direct Pore Matching for fingerprint Recognition. In: Proc. of Third International Conf. on Advances in Biometrics, Springer-Verlog, 2009, 797 - 606.

[107] Zhou Shang-Ming, Can John Q, Xu Li et al. Interactive Image Enhancement by Fuzzy Relaxation. International Journal of Automation and Computing, Springer,2007, 4(3): 229 - 235.

[108]Zhu Le-ging, Zhang San-yuan. Multimodal Biometric Identification System Based on Finger Geometry. Pattern Recognition Letters, Elsevier, 2010, 31(12): 1641 - 1649.

[109] Zhu Yong, Tan Tienuiu, Wang Yunhong. Biometric Personal Identification Based on Iris patterns. In: Proc. of IEEE fifteenth International Conf. on Pattern Recognition, 2000, 2: 801 - 804.

[110] Ziv J., Lempel A. A universal algorithm for sequential data compression. IEEE Transactions on Information Theory, 1977, 23(3): 337-343.

[111] Ziv J., Lempel A. Compression of Individual Sequences via Variable Rate Coding. IEEE Transactions on Information Theory, 1978, 24(5): 530-536.

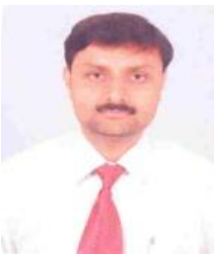

Prakash Chandra Srivastava received a Master degree in mathematics from University of Allahabad, INDIA in 2001, and Ph.D. degree from University of Allahabad, INDIA in 2009. He is an Assistant Professor at Birla Institute of Technology Ranchi, INDIA (Allahabad Campus). His research interest includes functional analysis and Software Measurement Methods.

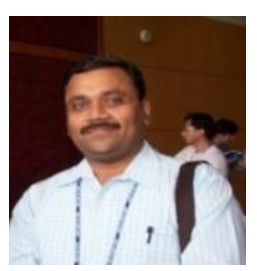

Anupam Agrawal is presently working as Professor of Computer Science and Information Technology at Indian Institute of Information Technology Allahabad (IIIT-A). Before joining IIIT-A in the year 2000, he was working as scientist 'D' at DEAL, DRDO, Govt. of India, Dehradun. He received his M.Tech. degree in Computer Science and Engineering from Indian Institute of Technology Madras, Chennai and Ph.D. degree in Information Technology from Indian Institute of Information Technology Allahabad (in association with Indian Institute of Technology, Roorkee). He was a postdoctoral researcher at the Department of Computer Science \& Technology, University of Bedfordshire (UK) during which he had contributed significantly in two major European projects. His research interests include Computer Vision, Image Processing, Visual Computing, Soft- Computing and Human-Computer Interaction. He has more than 75 publications related to these areas in various international journals and conference proceedings, and has co-authored one book. He is on the review board for various international journals including IEEE, Springer, MDPI, Taylor \& Franc is and Elsevier. He is currently serving as a Principal Investigator of an international (Indo-UK) Project. He is a member of ACM (USA), senior member of IEEE (USA) and a fellow of IETE (India). He is also serving as Chairman of the ACM Chapter at IIIT-A.

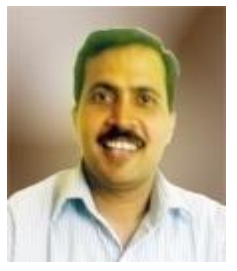

Kamta Nath Mishra received a Master of Technology (M.Tech., Software Systems) degree from Birla Institute of Technology and Science (BITS) Pilani, INDIA in 2003, and Master of Computer Application (MCA) degree from Madan Mohan Malviya Engineering College Gorakhpur, U.P., INDIA in 1996. He is an Assistant professor at Birla Institute of Technology, Mesra, Ranchi, INDIA (Allahabad Campus) and pursuing Ph.D. from the same Institute. Previously, Mr. Mishra worked with Sebha University, Libya, B.I.T. Noida, CDAC Noida, and K.I.E.T. Ghaziabad before joining B.I.T. Allahabad campus. His research interest includes Biometric Systems Based 
Personal Identification Methods, Image Processing and Analysis of Algorithms.

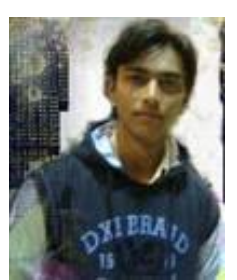

Pradeep Kumar Ojha received a Bachelor of Computer Application (B.C.A) degree from Dr. R.M.L. Awadh University Faizabad, INDIA in 2009 and he is currently pursuing Master of Computer Applications (MCA) degree from Birla Institute of Technology, Ranchi, INDIA (Allahabad Campus). His research interest includes Image Processing and personal identification methods.

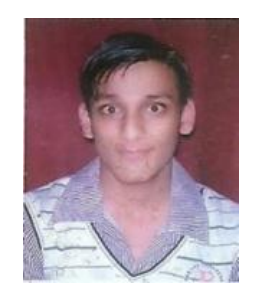

Rishu Garg received a Bachelor of Computer Application (BCA) degree from Rohilkhand University Bareilly, INDIA in 2010 and he is currently pursuing Master of Computer Applications (MCA) degree from Birla Institute of Technology, Ranchi, INDIA (Allahabad Campus). His research interest includes voice based identifications methods and Image Processing.

How to cite this paper: Prakash Chandra Srivastava, Anupam Agrawal, Kamta Nath Mishra, P. K. Ojha, R. Garg,"Fingerprints, Iris and DNA Features based Multimodal Systems: A Review", International Journal of Information Technology and Computer Science(IJITCS), vol.5, no.2, pp.88-111, 2013.DOI: $10.5815 /$ ijitcs.2013.02.10 